\title{
Levi equation for almost complex structures
}

\section{Giovanna Citti and Giuseppe Tomassini}

\begin{abstract}
In this paper we are dealing with the boundary problem for Levi flat graphs in the space $\mathbb{R}^{4}$, endowed with an almost complex structure $J$. This problem can be formalized as a Dirichlet problem for a quasilinear degenerate elliptic equation, called Levi equation. The Levi equation has the form

$$
D_{1}^{2}+D_{2}^{2}-D_{1} f=0
$$

where $D_{1}$ and $D_{2}$ are nonlinear vector fields. Under geometrical assumptions on the boundary a lipschitz continuous viscosity solution is found. The regularity of the viscosity solution is studied in suitable anisotropical Sobolev spaces, and it is proved that the solution has derivatives of any order in the direction of the vectors $D_{1}$ and $D_{2}$ i.e. it is of class $C^{\infty}$ in these directions, but not necessary regular in the third direction of the space. Finally, after proving a weak version of the Frobenius theorem, we show that the graph of the solution is foliated in holomorphic curves.
\end{abstract}

\section{Introduction and generalities}

Let $\left(\mathbb{R}^{4}, J\right)$ be the space $\mathbb{R}^{4}$ equipped with an almost complex structure $J$. We recall that $J$ is a differentiable map $\mathbb{R}^{4} \rightarrow G L(4, \mathbb{R})$ such that $J(p)^{2}=$ $-I d$, for every $p \in \mathbb{R}^{4}$. Let $M$ be a differentiable hypersurface in $\mathbb{R}^{4}$. For every $p \in M$ the tangent hyperplane $T_{p} M$ contains a (unique) $J$-invariant plane $T_{p}^{J} M$. The distribution of planes $p \mapsto T_{p}^{J} M$ is called the Levi distribution on $M$, and $M$ is said to be $J$-Levi flat whenever $p \mapsto T_{p}^{J} M$ is integrable.

2000 Mathematics Subject Classification: 35B65, 35H10, 32Q60, 58J.

Keywords: Levi equation, almost complex structure, degenerate elliptic equation, anysotropic Sobolev spaces, foliation in holomorphic curves. 
In view of Frobenius Theorem $M$ is then foliated by regular surfaces on which $J$ induces an integrable almost complex structure. Consequently $M$ is foliated by complex curves, whose complex structure is in general different from that induced by $J_{0}$, the standard one.

Let $J=J_{0}$. The problem of finding a Levi flat hypersurface with a prescribed boundary $\Gamma$ has been extensively studied by methods of the geometric theory of several complex variables (cf. $[\mathrm{BG}],[\mathrm{BK}],[\mathrm{A}],[\mathrm{S}],[\mathrm{K}]$, [CS], [ST]).

A different approach is found in [SIT] where the boundary problem for Levi flat graphs is reduced to a Dirichlet problem for a nonlinear, second order, elliptic degenerate operator $\mathcal{L}$, the so called Levi operator (see (1.7) below). Also an existence and uniqueness theorem of viscosity solutions was proved. Then, based on ideas from [FS], [RS] and [FL], a new regularization technique for nonlinear operators was settled down in [CM1], [CM2], [CLM], which allows to establish interior regularity of a (viscosity) solution $u$ of $\mathcal{L} u=0$ in the directions of non degeneracy. As a consequence, in [CM2] was proved that the graph of $u$ is foliated by holomorphic curves: a local result which is independent of the properties of the boundary $\Gamma$.

In the context of general almost complex structures Gromov in $[\mathrm{G}]$ proved a remarkable existence theorem under the following hypothesis:

a) $\Gamma$ is embedded in the boundary $b V$ of an almost complex manifold $(V, J)$ with no rational curve; $b V$ is strongly $J$-pseudoconvex and $J$ is a tamed almost complex structure. (Recall that an almost complex structure $J$ is said to be tamed by the symplectic form $\omega$ if $\omega\left(X, J_{p} X\right)>0$ for every $\left.X, Y \in \mathbb{R}_{p}^{4}, p \in \mathbb{R}^{4}\right)$.

b) $\Gamma$ is a smooth 2 -sphere with two only elliptic complex tangency points.

In the present paper we apply the methods of [SIT] and [CM2] to treat the boundary problem in a meaningful case which is not covered by Gromov's theorem. Precisely we consider a bounded domain $\Omega$ in the $\left(x_{1}, x_{2}, x_{3}\right)$-space and the (tamed by the standard symplectic form) almost complex structure $J$ on $\Omega \times \mathbb{R}$ defined by the matrix

$$
\left(\begin{array}{cccc}
0 & -1 & 0 & 0 \\
1 & 0 & 0 & 0 \\
f & 0 & 0 & -1 \\
0 & -f & 1 & 0
\end{array}\right)
$$

with $f$ regular on a neighbourhood of $\bar{\Omega} \times \mathbb{R}$. The boundary $\Gamma$ is a graph over $b \Omega$ for which we do not require the validity of the crucial condition b) in Gromov's theorem. 
We then prove that, under appropriate hypothesis for $\Gamma$ and $f$, there exists a $J$-Levi flat graph $M$ over $\Omega$ whose boundary is $\Gamma$ (cf. Th. 1.1).

Let us make explicit the analytic condition in order for a graph $M=$ $\left\{x_{4}=u\left(x_{1}, x_{2}, x_{3}\right)\right\}$ to be $J$-Levi flat. It is easily obtained, for the $J$-invariant tangent plane $T_{p}^{J} M$ at a point $p \in M$ is spanned by the vectors

$$
\xi=(1,0, a, f-b) \quad \text { and } \quad \eta=(0,1, b, a)
$$

where

$$
a=a(u)=\frac{\partial_{2} u-\partial_{3} u\left(\partial_{1} u-f\right)}{1+\left(\partial_{3} u\right)^{2}}, \quad b=b(u)=-\frac{\partial_{2} u \partial_{3} u+\partial_{1} u-f}{1+\left(\partial_{3} u\right)^{2}} .
$$

Let $D_{1}$ and $D_{2}$ denote the vector fields

$$
D_{1}=\partial_{1}+a \partial_{3}, \quad D_{2}=\partial_{2}+b \partial_{3}
$$

then $\xi=D_{1}+(f-b) \partial_{4}, \eta=D_{2}+a \partial_{4}$ and $[\xi, \eta]=0$ if and only if $\left[D_{1}, D_{2}\right]=0$. $D_{1}$ and $D_{2}$ satisfy the following main identity:

$$
D_{1} u=-b+f, \quad D_{2} u=a
$$

(see [CM1] for the case $f=0$ ). Evaluating the bracket $\left[D_{1}, D_{2}\right]$, we then obtain

$$
\left[D_{1}, D_{2}\right]=\left(D_{1} b-D_{2} a\right) \partial_{3}=-\left(D_{1}^{2} u+D_{2}^{2} u-D_{1} f\right) \partial_{3} .
$$

In particular the graph of $u$ is J-Levi flat if and only if $u$ is a solution of

$$
\mathcal{L}^{J} u=D_{1}^{2} u+D_{2}^{2} u-D_{1} f=0 .
$$

$\mathcal{L}^{J} u$ is, by definition, the $J$-Levi operator for the almost complex structure $J . \mathcal{L}^{J} u$ is a second order elliptic degenerate operator with two positive eigenvalues. Thus, in our situation, the boundary problem for $J$-Levi flat hypersurfaces amounts to solve the Dirichlet problem

$$
\begin{cases}\mathcal{L}^{J} u=0 & \text { in } \quad \Omega \\ u=g & \text { on } \quad b \Omega .\end{cases}
$$

The main result of the paper is contained in the following

Theorem 1.1 Assume that $\Omega$ is bounded and $b \Omega$ is strictly $J$-pseudoconvex. Let $f \in C^{m+1}(\bar{\Omega}), g \in C^{2}(b \Omega)$ and either $f=0$ or $\sup \partial_{1} f<0$ in $\bar{\Omega}$. Then the problem (1.8) has a unique (viscosity) solution $u \in \operatorname{Lip}(\bar{\Omega})$ whose Lie derivatives of order $k \leq m$, in the directions of the vector fields $D_{i}$, are of class $C_{\text {loc }}^{\alpha}$ for all $\alpha<1$. u may not be regular in the usual sense but its graph is foliated by complex curves. 
In order to prove the main theorem we approximate (1.8) by the problem

$$
\begin{cases}\mathcal{L}_{\varepsilon}^{J} u=0 & \text { in } \quad \Omega \\ u=g & \text { on } b \Omega\end{cases}
$$

where

$$
\mathcal{L}_{\varepsilon}^{J}=D_{1}^{2}+D_{2}^{2}+D_{3}^{2}-D_{1} f
$$

and

$$
D_{3}=\frac{\varepsilon}{\sqrt{1+\left(\partial_{3} u\right)^{2}}} \partial_{3}, \quad \varepsilon>0 .
$$

The existence of a regular solution to (1.9) is proved in Section 2. In our context the operators $D_{i}$ play the same rôle as the derivatives in the classical setting. So under the assumption that $b \Omega$ is strictly $J$-pseudoconvex, we establish some a priori uniform (with respect to $\varepsilon$ ) estimates for $\partial_{3} u$ and for the intrinsic gradient

$$
\left(D_{1} u, D_{2} u, D_{3} u\right)
$$

(cf. Th. 2.7). We observe that, due to the presence of $f$, the proof of these estimates is technically much more involved than in the case of the standard complex structure $J_{0}$ (when $f=0$ ). Once the gradient estimate is achieved, the existence of a regular solution $u_{\varepsilon}$ of (1.9) classically follows. We also immediately deduce that $u=\lim _{\varepsilon \rightarrow 0} u_{\varepsilon}$ is a $\operatorname{Lip}(\bar{\Omega})$ solution of (1.8) (cf. Th. 4.1).

Next, following [CLM], [CM2], natural anisotropic Sobolev spaces are defined, (cf. Sec. 3). Using in full strength the representation (1.10) of $\mathcal{L}_{\varepsilon}^{J}$ and the technique of [CLM], [CM2], a priori uniform estimates in this setting are proved for $u_{\varepsilon}$ and $u$ (cf. Cor. 3.6 and Th. 4.3).

Finally, in the last section we show that the graph of $u$ is foliated by holomorphic curves. To this end we study the existence of Lie derivatives in the intrinsic directions $D_{1}, D_{2}$. We emphasize that here we cannot apply the same technique as in the case $f=0$ where the local regularity of $u$ was consequence of some analytic estimates. In our situation we have not such estimates, $f$ being not analytic, so we cannot argue in the same way. Instead we prove a weak version of Frobenius Theorem with non Lipschitz coefficients:

Theorem 1.2 Let $\theta, \zeta \in C_{\text {loc }}^{\alpha}, \alpha>1 / 2$, and $\widetilde{D}_{1}=\partial_{1}+\theta \partial_{3}, \widetilde{D}_{2}=\partial_{1}+\zeta \partial_{3}$. Assume that the distributional derivatives $\widetilde{D}_{i} \theta, \widetilde{D}_{i} \zeta$ exist and belong to $C_{\text {loc }}^{\alpha}(\Omega)$ and that $\partial_{3} \theta, \partial_{3} \zeta \in L_{\mathrm{loc}}^{p}(\Omega), p>3 n$. Then, if the compatibility conditon $D_{1} \zeta=D_{2} \theta$ is fullfilled, in a neighbourhood of a fixed $\bar{\xi}=\left(\bar{x}_{1}, \bar{x}_{2}\right)$ there exists a local solution $t \in C^{\alpha}$ of the system

$$
\left\{\begin{array}{l}
\partial_{1} t\left(x_{1}, x_{2}\right)=\theta\left(x_{1}, x_{2}, t\left(x_{1}, x_{2}\right)\right) \\
\partial_{2} t\left(x_{1}, x_{2}\right)=\zeta\left(x_{1}, x_{2}, t\left(x_{1}, x_{2}\right)\right)
\end{array}\right.
$$

Then the foliation property follows (cf. prof. of Th. 1.1). 


\section{Existence theorem for elliptic regularization}

In this section we find a solution of the regularized Dirichlet problem (1.9). We suitably adapt the classical procedure for quasilinear equations to our context. Using the usual representation of $\mathcal{L}^{J} u$ in coordinates, we establish a priori bounds for $u$ and its gradient at the boundary. Then, using the particular structure of the operator as a sum of squares, we establish an a priori bound for the intrinsic gradient $\left(D_{1} u, D_{2} u, D_{3} u\right)$, and for $\partial_{3} u$, instead of the standard gradient. This procedure leads in particular to an a priori bound for the gradient, and the existence of a solution classically follows.

\subsection{A priori bound for $u$}

Let us represent the operator $\mathcal{L}^{J}$ in coordinates.

Proposition 2.1 The operator $\mathcal{L}^{J} u$ writes

$$
\mathcal{L}^{J} u=\frac{\widetilde{\mathcal{L}}^{J} u}{1+\left(\partial_{3} u\right)^{2}}
$$

where

$$
\begin{gathered}
\widetilde{\mathcal{L}}^{J} u=\partial_{11} u+\partial_{22} u+2 a \partial_{13} u+2 b \partial_{23} u+\left(a^{2}+b^{2}\right) \partial_{33} u- \\
-\left(\partial_{3} f \partial_{2} u-\partial_{2} f \partial_{3} u+\partial_{1} f\right) .
\end{gathered}
$$

Proof. By definition

$$
\begin{aligned}
& \mathcal{L}^{J} u=\left(\partial_{1}+a \partial_{3}\right)^{2} u+\left(\partial_{2}+b \partial_{3}\right)^{2} u-D_{1} f \\
& =\partial_{11} u+2 a \partial_{13} u+a^{2} \partial_{33} u+D_{1} a \partial_{3} u+\partial_{22} u+2 b \partial_{23} u+b^{2} \partial_{33} u+D_{2} b \partial_{3} u-D_{1} f .
\end{aligned}
$$

Clearly, we have only to compute

$$
D_{1} a \partial_{3} u+D_{2} b \partial_{3} u-D_{1} f=D_{1} D_{2} \partial_{3} u-D_{2} D_{1} u \partial_{3} u+D_{2} f \partial_{3} u-D_{1} f=
$$

(since $\left[D_{1}, D_{2}\right] u=-\mathcal{L}^{J} u \partial_{3} u$ )

$$
=-\mathcal{L}^{J} u\left(\partial_{3} u\right)^{2}+\partial_{2} f \partial_{3} u+b \partial_{3} f \partial_{3} u-\partial_{1} f-a \partial_{3} f=
$$

(since $b \partial_{3} u-a=b \partial_{3} u-D_{2} u=-\partial_{2} u$ )

$$
=-\mathcal{L}^{J} u\left(\partial_{3} u\right)^{2}+\partial_{2} f \partial_{3} u-\partial_{2} u \partial_{3} f-\partial_{1} f .
$$

Inserting this in the previous expression we get the assertion. 
Let us also note that for every derivation $X$ one has

$$
X D_{3} u=\frac{\varepsilon}{\left(1+\left(\partial_{3} u\right)^{2}\right)^{3 / 2}} X \partial_{3} u .
$$

Then the elliptic regularisation (1.10) of $\mathcal{L}^{J} u$ can be expressed as follows:

$$
\mathcal{L}_{\varepsilon}^{J} u=\mathcal{L}^{J} u+D_{3}^{2} u=\frac{1}{1+\left(\partial_{3} u\right)^{2}}\left(\widetilde{\mathcal{L}}^{J} u+\frac{\varepsilon^{2}}{1+\left(\partial_{3} u\right)^{2}} \partial_{3}^{2} u\right),
$$

and we call

$$
\widetilde{\mathcal{L}}_{\varepsilon}^{J} u=\widetilde{\mathcal{L}}^{J} u+\frac{\varepsilon^{2}}{1+\left(\partial_{3} u\right)^{2}} \partial_{3}^{2} u
$$

the elliptic regularisation for $\widetilde{\mathcal{L}}^{J} u$. Since $\mathcal{L}_{\varepsilon}^{J}$ is elliptic, for a regular function $u \in C^{0}(\bar{\Omega})$ we have the following:

1) if $\partial_{1} f \geq 0$ and $\mathcal{L}_{\varepsilon}^{J}(u) \geq 0$ then $u(x) \leq \max _{b \Omega} u$;

2) if $\partial_{1} f \leq 0$ and $\mathcal{L}_{\varepsilon}^{J}(u) \leq 0$ then $\min _{b \Omega} u \leq u(x)$

for every $x \in \bar{\Omega}$.

Let us now prove a comparison principle for subsolutions and supersolutions. Consider two regular functions $u, v$ such that $\mathcal{L}_{\varepsilon}^{J}(u) \geq \mathcal{L}_{\varepsilon}^{J}(v)$ and let $w=u-v$. We have

$$
\begin{aligned}
\left(1+\left(\partial_{3} v\right)^{2}\right) & \left\{\mathcal{L}_{\varepsilon}^{J}(u)-\mathcal{L}_{\varepsilon}^{J}(v)\right\}=\widetilde{\mathcal{L}}_{\varepsilon}^{J}(u)-\widetilde{\mathcal{L}}_{\varepsilon}^{J}(v)+\sum_{j=1}^{3} \widetilde{c}_{j} \partial_{j} w \\
= & \partial_{11} w+\partial_{22} w+2 a \partial_{13} w+2 b \partial_{23} w+\left(a^{2}+b^{2}\right) \partial_{33} w+ \\
& +\frac{\varepsilon^{2}}{1+\left(\partial_{3} u\right)^{2}} \partial_{3}^{2} w+\sum_{j=1}^{3} \widetilde{c}_{j} \partial_{j} w=\mathcal{Q}_{\varepsilon}^{J}(w),
\end{aligned}
$$

where $a=a(u), b=b(u)$ and $\widetilde{c}_{j}=\widetilde{c}_{j}(u, v)$ is linear in $\varepsilon^{2}$. Since $\mathcal{Q}_{\varepsilon}^{J}(w)$ is elliptic, then

$$
(u-v)(x) \leq \max _{b \Omega}(u-v)
$$

for every $x \in \bar{\Omega}$.

Now take $\alpha, \beta$ such that $\left(x_{1}-\alpha\right)^{2}+\left(x_{2}-\beta\right)^{2}>0$ in $\bar{\Omega}$ and consider the function $v^{-}=\exp c\left(\left(x_{1}-\alpha\right)^{2}+\left(x_{2}-\beta\right)^{2}\right)$. We have

$$
\begin{aligned}
\widetilde{\mathcal{L}}_{\varepsilon}^{J}\left(v^{-}\right)= & \exp c\left(\left(x_{1}-\alpha\right)^{2}+\left(x_{2}-\beta\right)^{2}\right)\left[4 c+4 c^{2}\left(\left(x_{1}-\alpha\right)^{2}+\left(x_{2}-\beta\right)^{2}\right)-\right. \\
& \left.-2 c\left(x_{2}-\beta\right) \partial_{2} f\right]-\partial_{1} f ;
\end{aligned}
$$

thus $\widetilde{\mathcal{L}}_{\varepsilon}^{J}\left(v^{-}\right)>0$ (uniformly with respect to $\varepsilon \rightarrow 0$ ) if $c$ is sufficiently large.

Similarly, if $v^{+}=-\exp c\left(\left(x_{1}-\alpha\right)^{2}+\left(x_{2}-\beta\right)^{2}\right)$, we have $\widetilde{\mathcal{L}}_{\varepsilon}^{J}\left(v^{+}\right)<0$. 
In view of the comparison principle, for a solution $u \in C^{2}(\Omega) \cap C^{1}(\bar{\Omega})$ of $\widetilde{\mathcal{L}}_{\varepsilon}^{J} u=0$ we derive the following estimates

$$
\begin{aligned}
& u(x)-v^{+}(x) \leq \max _{b \Omega}\left(u-v^{+}\right) \\
& v^{-}(x)-u(x) \leq \max _{b \Omega}\left(v^{-}-u\right) .
\end{aligned}
$$

In particular we obtain the a priori estimate

$$
\|u\|_{C^{0}(\bar{\Omega})}<C
$$

where $C$ is a constant depending only on $u_{\mid b \Omega}$.

\subsection{A priori bound for $\nabla u$ at the boundary}

As usual we first estimate the normal derivative and next we bound $|\nabla u|$ by a constant $C$ depending only on $\nabla u_{\mid b \Omega}$.

Let us suppose that $\Omega$ is defined by $\varrho<0$ where $\varrho$ is a smooth function such that $\nabla \varrho \neq 0$ on $b \Omega$. Let $g$ be in $C^{2}(b \Omega)$ and $\widetilde{g}$ be a $C^{2}$ extension of $g$ to $\mathbb{R}^{3}$. Let $u^{-}=\widetilde{g}+\lambda \varrho, u^{+}=\widetilde{g}-\lambda \varrho$ where $\lambda$ is a positive constant. We have

$$
\begin{gathered}
\left(1+\left(\partial_{3} u^{\mp}\right)^{2}\right) \widetilde{\mathcal{L}}_{\varepsilon}^{J}\left(u^{\mp}\right)= \pm \lambda^{3}\left\{\left(\partial_{3} \varrho\right)^{2}\left(\partial_{11} \varrho+\partial_{22} \varrho\right)+\left(\left(\partial_{1} \varrho\right)^{2}+\left(\partial_{2} \varrho\right)^{2}\right) \partial_{33} \varrho-\right. \\
\left.-2 \partial_{1} \varrho \partial_{3} \varrho \partial_{13} \varrho-2 \partial_{2} \varrho \partial_{3} \varrho \partial_{23} \varrho+\left(\partial_{3} \varrho\right)^{2}\left(\partial_{2} \varrho \partial_{3} f+\partial_{3} \varrho \partial_{2} f\right)\right\}+R(\lambda)
\end{gathered}
$$

where $R(\lambda)$ is a polynomial in $\lambda$ of degree $\leq 2$ whose coefficients are uniformly bounded as $\varepsilon \rightarrow 0$. Let us define the function $k^{J}: b \Omega \rightarrow \mathbb{R}$ by

$$
\begin{aligned}
k^{J}= & \left(\partial_{3} \varrho\right)^{2}\left(\partial_{11} \varrho+\partial_{22} \varrho\right)+\left(\left(\partial_{1} \varrho\right)^{2}+\left(\partial_{2} \varrho\right)^{2}\right) \partial_{33} \varrho-2 \partial_{1} \varrho \partial_{3} \varrho \partial_{13} \varrho- \\
& -2 \partial_{2} \varrho \partial_{3} \varrho \partial_{23} \varrho+\left(\partial_{3} \varrho\right)^{2}\left(\partial_{2} \varrho \partial_{3} f+\partial_{3} \varrho \partial_{2} f\right) .
\end{aligned}
$$

The condition $k^{J}>0$ on $b \Omega$ does not depend on the defining function $\varrho$ and provides the notion of $J$-pseudoconvexity of $b \Omega$. Thus, if $b \Omega$ is strictly $J$-pseudoconvex, $\widetilde{\mathcal{L}}_{\varepsilon}^{J}\left(u^{-}\right)>0$ and $\widetilde{\mathcal{L}}_{\varepsilon}^{J}\left(u^{+}\right)<0$, provided $\lambda$ is sufficiently large.

Since $u^{ \pm}=g$ on $b \Omega$ in view of the comparison principle we obtain the following: if $u$ is a regular solution of the Dirichlet problem (1.9) then

$$
u^{-} \leq u \leq u^{+}
$$

In particular for the normal derivative $\partial u / \partial \nu$ we have the estimate

$$
\|\partial u / \partial \nu\|_{b \Omega}<C
$$

where $C$ is a positive constant depending only on $\nabla g$ and $\nabla^{2} g$. 


\subsection{A priori bound for $\nabla u$ in $\Omega$}

We proceed now to prove an a priori estimate for $D u$ treating $\left(\partial_{3} u\right)^{2}$ and $\left(D_{1} u\right)^{2}+\left(D_{2} u\right)^{2}$ separately, $\left\{\partial_{3}, D_{1}, D_{2}\right\}$ being a base of $T_{x} \mathbb{R}^{3}$.

The proof is actually rather involved and requires some technical, preparatory results, which are collected in the following three lemmas.

Lemma 2.2 Let $u$ be a regular function $a$ and $b$ the associated coefficients defined in (1.3). We have the identities:

$$
\begin{aligned}
\partial_{3} b & =-\frac{D_{1} \partial_{3} u+\partial_{3} u D_{2} \partial_{3} u}{1+\left(\partial_{3} u\right)^{2}}+\frac{\partial_{3} f}{1+\left(\partial_{3} u\right)^{2}} \\
\partial_{3} a & =\frac{D_{2} \partial_{3} u-\partial_{3} u D_{1} \partial_{3} u}{1+\left(\partial_{3} u\right)^{2}}+\frac{\partial_{3} u \partial_{3} f}{1+\left(\partial_{3} u\right)^{2}} .
\end{aligned}
$$

Proof. These two assertions are similar so we give here only the proof of the first one. We argue as in [CM1] (for the case $f=0$ ).

Since $\left[\partial_{3}, D_{1}\right]=\partial_{3} a \partial_{3},\left[\partial_{3}, D_{2}\right]=\partial_{3} b \partial_{3}$ from (1.5) we derive

$$
\partial_{3} b=-\partial_{3} D_{1} u+\partial_{3} f=-\partial_{3} a \partial_{3} u-D_{1} \partial_{3} u+\partial_{3} f
$$

again by (1.5) the last quantity equals

$$
-\partial_{3} D_{2} u \partial_{3} u-D_{1} \partial_{3} u+\partial_{3} f=-\partial_{3} b\left(\partial_{3} u\right)^{2}-D_{2} \partial_{3} u \partial_{3} u-D_{1} \partial_{3} u+\partial_{3} f .
$$

The assertion follows immediately.

Lemma 2.3 Let $u$ be a regular solution of $\mathcal{L}_{\varepsilon}^{J} u=0$ and $v=\arctan \left(\partial_{3} u\right)$. Then for the brackets of $D_{1}, D_{2}, D_{3}$ we have the following formulas:

$$
\begin{aligned}
& {\left[D_{1}, D_{2}\right]=D_{3} v D_{3}, \quad\left[D_{1}, D_{3}\right]=-D_{2} v D_{3}-\frac{\partial_{3} f}{1+\left(\partial_{3} u\right)^{2}} \partial_{3} u D_{3}} \\
& {\left[D_{2}, D_{3}\right]=D_{1} v D_{3}-\frac{\partial_{3} f}{1+\left(\partial_{3} u\right)^{2}} D_{3} .}
\end{aligned}
$$

Proof. Since $\mathcal{L}_{\varepsilon}^{J} u=0$, the first identity immediately follows from (1.6) for

$$
\left[D_{1}, D_{2}\right]=-\left(D_{1}^{2} u+D_{2}^{2} u-D_{1} f\right) \partial_{3}=D_{3}^{2} u \partial_{3}
$$

and $D_{3}^{2} u \partial_{3}=D_{3} v D_{3}$ by virtue of $(2.2)$.

As for the second, in view of (2.7), we have

$$
\begin{aligned}
{\left[D_{1}, D_{3}\right] } & =\left(D_{1}\left(\frac{\varepsilon}{\sqrt{1+\left(\partial_{3} u\right)^{2}}}\right)-D_{3} a\right) \partial_{3}= \\
& =-\left(\partial_{3} u \frac{D_{1} \partial_{3} u}{1+\left(\partial_{3} u\right)^{2}}+\partial_{3} a\right) \frac{\varepsilon \partial_{3}}{\sqrt{1+\left(\partial_{3} u\right)^{2}}}=-\frac{D_{2} \partial_{3} u+\partial_{3} u \partial_{3} f}{1+\left(\partial_{3} u\right)^{2}} D_{3} .
\end{aligned}
$$

The proof of the third assertion is similar. 
Lemma 2.4 Let $\theta$ be a regular function and $w$ a regular solution of

$$
\sum_{j=1}^{3} D_{j}^{2} w=\theta .
$$

Then

$$
\begin{aligned}
\sum_{j=1}^{3} D_{j}^{2}\left(D_{1} w\right)= & D_{1} \theta-\frac{2 D_{3} \partial_{3} u D_{23} w}{1+\left(\partial_{3} u\right)^{2}}+\frac{2 D_{2} \partial_{3} u D_{3}^{2} w}{1+\left(\partial_{3} u\right)^{2}}+ \\
& +2 \frac{\partial_{3} u \partial_{3} f}{1+\left(\partial_{3} u\right)^{2}} D_{3}^{2} w+D_{3}\left(\frac{\partial_{3} u \partial_{3} f}{1+\left(\partial_{3} u\right)^{2}}\right) D_{3} w
\end{aligned}
$$

$$
\begin{aligned}
\sum_{j=1}^{3} D_{j}^{2}\left(D_{2} w\right)= & D_{2} \theta+\frac{2 D_{3} \partial_{3} u D_{13} w}{1+\left(\partial_{3} u\right)^{2}}-\frac{2 D_{1} \partial_{3} u D_{3}^{2} w}{1+\left(\partial_{3} u\right)^{2}} \\
& +2 \frac{\partial_{3} f}{1+\left(\partial_{3} u\right)^{2}} D_{3}^{2} w+D_{3}\left(\frac{\partial_{3} f}{1+\left(\partial_{3} u\right)^{2}}\right) D_{3} w
\end{aligned}
$$

$$
\begin{aligned}
\sum_{j=1}^{3} D_{j}^{2}\left(\partial_{3} w\right)= & \partial_{3} \theta-\partial_{3} D_{2} f \partial_{3} w-2 \partial_{3} a D_{1} \partial_{3} w- \\
& -2 \partial_{3} b D_{2} \partial_{3} w+2 \partial_{3} u D_{3} v D_{3} \partial_{3} w
\end{aligned}
$$

Moreover

$$
\partial_{3}\left(D_{2}(f)\right) \partial_{3} w=\partial_{3}\left(\partial_{2} f-D_{1} u \partial_{3} f+f \partial_{3} f\right) \partial_{3} w
$$

where $v=\arctan \left(\partial_{3} u\right)$.

Proof. Set $s_{1}=D_{1} w$. Differentiating the equation with respect to $D_{1}$ and using the identities (2.8) we get

$$
\begin{aligned}
D_{1}^{2} s_{1}+ & D_{1}^{2} s_{1}+D_{3}^{2} s_{1}= \\
= & D_{1} \theta-\left[D_{1}, D_{2}\right] D_{2} w-D_{2}\left[D_{1}, D_{2}\right] w-\left[D_{1}, D_{3}\right] D_{3} w-D_{3}\left[D_{1}, D_{3}\right] w= \\
= & D_{1} \theta-D_{3} v D_{3} D_{2} w-D_{2} D_{3} v D_{3} w+D_{2} v D_{3}^{2} w+ \\
& +\frac{\partial_{3} f}{1+\left(\partial_{3} u\right)^{2}} \partial_{3} u D_{3}^{2} w+D_{3}\left(D_{2} v D_{3} w\right)+D_{3}\left(\frac{\partial_{3} u \partial_{3} f}{1+\left(\partial_{3} u\right)^{2}} D_{3} w\right) .
\end{aligned}
$$

Observe that, by virtue of (2.8)

$$
\begin{aligned}
& -D_{3} v D_{3} D_{2} w+D_{2} v D_{3}^{2} w=-D_{3} v D_{2} D_{3} w+D_{1} v D_{3} v D_{3} w+D_{2} v D_{3}^{2} w= \\
& =-D_{2}\left(D_{3} v D_{3} w\right)+D_{2} D_{3} v D_{3} w+D_{1} v D_{3} v D_{3} w+D_{3}\left(D_{2} v D_{3} w\right)-D_{3} D_{2} v D_{3} w \\
& =-D_{2}\left(D_{3} v D_{3} w\right)+D_{3}\left(D_{2} v D_{3} w\right)+2 D_{1} v D_{3} v D_{3} w .
\end{aligned}
$$

Substituting this in the last equality we obtain the first assertion. 
The proof of the second identity is similar.

As for the third set $s_{3}=\partial_{3} w, \zeta=\left(\left(1+\left(\partial_{3} u\right)^{2}\right)^{-1 / 2}\right.$ and differentiate the equation with respect to $\partial_{3}$. We get

$$
\sum_{i=1}^{3} D_{i}^{2} s_{3}=\sum_{i=1}^{3}\left(D_{i} \theta-\left[\partial_{3}, D_{i}\right] D_{i} w-D_{i}\left[D_{i}, \partial_{3}\right] w\right) .
$$

Let us compute the terms which contain the commutators:

$$
\begin{gathered}
{\left[\partial_{3}, D_{i}\right]\left(D_{i} w\right)+D_{i}\left(\left[D_{i}, \partial_{3}\right] w\right)=} \\
=\partial_{3} a \partial_{3} D_{1} w+D_{1}\left(\partial_{3} a \partial_{3} w\right)+\partial_{3} b \partial_{3} D_{2} w+D_{2}\left(\partial_{3} b \partial_{3} w\right)+\partial_{3} \zeta \partial_{3} D_{3} w+D_{3}\left(\partial_{3} \zeta \partial_{3} w\right) \\
=\partial_{3} a D_{1} \partial_{3} w+\partial_{3} a\left[\partial_{3}, D_{1}\right] w+\left[D_{1}, \partial_{3}\right] a \partial_{3} w+\partial_{3} D_{1} a \partial_{3} w+\partial_{3} a D_{1} \partial_{3} w+ \\
+\partial_{3} b D_{2} \partial_{3} w+\partial_{3} b\left[\partial_{3}, D_{2}\right] w+\left[D_{2}, \partial_{3}\right] b \partial_{3} w+\partial_{3} D_{2} b \partial_{3} w+\partial_{3} b D_{2} \partial_{3} w+ \\
+\partial_{3} \zeta D_{3} \partial_{3} w+\partial_{3} \zeta\left[\partial_{3}, D_{3}\right] w+\left[D_{3}, \partial_{3}\right] \zeta \partial_{3} w+\partial_{3} D_{3} \zeta \partial_{3} w+\partial_{3} \zeta D_{3} \partial_{3} w \\
=2\left(\partial_{3} a D_{1} \partial_{3} w+\partial_{3} b D_{2} \partial_{3} w+\partial_{3} u D_{3} v \partial_{3} w\right)+\partial_{3}\left(D_{1} a+D_{2} b+D_{3} \zeta\right) \partial_{3} w
\end{gathered}
$$

since $\partial_{3} a\left[\partial_{3}, D_{1}\right] \omega+\left[D_{1}, \partial_{3}\right] a \partial_{3} \omega$ vanishes, as well as the analogous terms involving $b$ and $\zeta$. In view of $(1.5)$ and $\left[D_{1}, D_{2}\right]=D_{3}^{2} u \partial_{3}$ from the last equality we derive

$$
\begin{aligned}
= & 2\left(\partial_{3} a D_{1} \partial_{3} w+\partial_{3} b D_{2} \partial_{3} w+\partial_{3} u D_{3} v \partial_{3} w\right)+ \\
& +\partial_{3}\left(D_{1} D_{2} u-D_{2} D_{1} u+D_{2} f-D_{3}^{2} u \partial_{3} u\right) \partial_{3} w= \\
= & 2\left(\partial_{3} a D_{1} \partial_{3} w+\partial_{3} b D_{2} \partial_{3} w+\partial_{3} u D_{3} v \partial_{3} w\right)+\partial_{3} D_{2} f \partial_{3} w .
\end{aligned}
$$

Now we are in position to prove the maximum principle for the gradient.

Proposition 2.5 Assume that $f \in C^{2}(\bar{\Omega})$ and that there exist constants $\alpha$ and $\beta$ such that

$$
-\alpha \partial_{1} f-\frac{\left|\partial_{13} f\right|}{\beta}-\frac{\left|\partial_{33} f\right|^{2}}{\alpha^{2}}\left(\frac{1}{\beta^{2}}+1\right)-\partial_{32} f-f \partial_{33} f-\left|\partial_{3} f\right|^{2} \geq 0 .
$$

Let $u \in C^{3}(\Omega) \cap C^{2}(\bar{\Omega})$ be a regular solution to (1.9). Then

$$
\left|\partial_{3} u\right| \leq C
$$

where $C$ is a constant only depending on $\max _{b \Omega}|\nabla u|$. 
Proof. Let us write the equation (1.9) in the form

$$
\sum_{j=1}^{3} D_{j}^{2} u=\partial_{1} f+D_{2} u \partial_{3} f
$$

Let $\psi$ be an invertible function, to be chosen later, such that $u=\psi(\bar{u})$. Then

$$
\begin{aligned}
& D_{j} u=\psi^{\prime}(\bar{u}) D_{j} \bar{u}, \\
& D_{j}^{2} u=\psi^{\prime}(\bar{u}) D_{j}^{2} \bar{u}+\psi^{\prime \prime}(\bar{u})\left(D_{j} \bar{u}\right)^{2}=\psi^{\prime}(\bar{u}) D_{j}^{2} \bar{u}+\bar{\eta}\left(D_{j} u\right)^{2},
\end{aligned}
$$

$j=1,2$, where $\bar{\eta}$ denotes the function $\psi^{\prime \prime} /\left(\psi^{\prime}\right)^{2}$. Substituting in (2.15) we obtain

$$
\sum_{j=1}^{3} D_{j}^{2} \bar{u}+\frac{\bar{\eta}}{\psi^{\prime}} \sum_{j=1}^{3}\left(D_{j} u\right)^{2}=\frac{1}{\psi^{\prime}}\left(\partial_{1} f+D_{2} u \partial_{3} f\right),
$$

and from this, applying $\partial_{3}$, in view of $(2.12)$, we deduce

$$
\begin{aligned}
\sum_{j=1}^{3} D_{j}^{2} \partial_{3} \bar{u}+ & \left(\frac{\bar{\eta}^{\prime}}{\psi^{\prime}}-\bar{\eta}^{2}\right) \sum_{j=1}^{3}\left(D_{j} u\right)^{2} \partial_{3} \bar{u}+2 \frac{\bar{\eta}}{\psi^{\prime}} \sum_{j=1}^{3} D_{j} u \partial_{3} D_{j} u= \\
= & \partial_{3}\left(\frac{\partial_{1} f+D_{2} u \partial_{3} f}{\psi^{\prime}}\right)-\partial_{3}\left(f_{2}-D_{1} u \partial_{3} f+f \partial_{3} f\right) \partial_{3} \bar{u}- \\
& -2 \partial_{3} a D_{1} \partial_{3} \bar{u}-2 \partial_{3} b D_{2} \partial_{3} \bar{u}+2 \partial_{3} u D_{3} v D_{3} \partial_{3} \bar{u}
\end{aligned}
$$

Let us denote $A_{1}, A_{2}, A_{3}$ the terms at the left hand side, and $A_{4}, \ldots, A_{8}$ those at the right one.

Since

$$
D_{j}^{2}\left(\left(\partial_{3} \bar{u}\right)^{2}\right)=D_{j}\left(2 \partial_{3} \bar{u} D_{j} \partial_{3} \bar{u}\right)=2\left(D_{j} \partial_{3} \bar{u}\right)^{2}+2 \partial_{3} \bar{u} D_{j}^{2} \partial_{3} \bar{u}
$$

then

$$
\partial_{3} \bar{u} A_{1}=\partial_{3} \bar{u} \sum_{j=1}^{3} D_{j}^{2} \partial_{3} \bar{u}=\frac{1}{2} \sum_{j=1}^{3} D_{j}^{2}\left(\left(\partial_{3} \bar{u}\right)^{2}\right)-\sum_{j=1}^{3}\left(D_{j} \partial_{3} \bar{u}\right)^{2} .
$$

Besides, using (1.5) and Lemma 2.2 to evaluate $\partial_{3} D_{1} u, \partial_{3} D_{2} u$, and (2.2) to compute $\partial_{3} D_{3} u$, we have

$$
\begin{aligned}
A_{3}= & 2 \frac{\bar{\eta}}{\psi^{\prime}}\left(D_{1} u \partial_{3} D_{1} u+D_{2} u \partial_{3} D_{2} u+D_{3} u \partial_{3} D_{3} u\right)= \\
= & 2 \frac{\bar{\eta}}{\psi^{\prime}} D_{1} u\left(\frac{D_{1} \partial_{3} u+\partial_{3} u D_{2} \partial_{3} u}{1+\left(\partial_{3} u\right)^{2}}+\frac{\left(\partial_{3} u\right)^{2}}{1+\left(\partial_{3} u\right)^{2}} \partial_{3} f\right)+ \\
& +2 \frac{\bar{\eta}}{\psi^{\prime}} D_{2} u\left(\frac{D_{2} \partial_{3} u-\partial_{3} u D_{1} \partial_{3} u}{1+\left(\partial_{3} u\right)^{2}}+\frac{\partial_{3} u}{1+\left(\partial_{3} u\right)^{2}} \partial_{3} f\right)+2 \frac{\bar{\eta}}{\psi^{\prime}} \frac{D_{3} u D_{3} \partial_{3} u}{1+\left(\partial_{3} u\right)^{2}} .
\end{aligned}
$$


Using (2.16) for $j=1,2,3$, we have

$$
\frac{D_{j} \partial_{3} u}{\psi^{\prime}}=\frac{D_{j}\left(\partial_{3} \bar{u} \psi^{\prime}\right)}{\psi^{\prime}}=D_{j} \partial_{3} \bar{u}+\frac{\psi^{\prime \prime}}{\psi^{\prime}} \partial_{3} \bar{u} D_{j} \bar{u}=D_{j} \partial_{3} \bar{u}+\bar{\eta} D_{j} u \partial_{3} \bar{u}
$$

so that, substituting in the expression of $A_{3}$, and using again (2.16), we have

$$
\begin{aligned}
& A_{3} \partial_{3} \bar{u}=2 \bar{\eta} D_{1} u\left(\frac{D_{1} \partial_{3} \bar{u}+\partial_{3} u D_{2} \partial_{3} \bar{u}}{1+\left(\partial_{3} u\right)^{2}}+\bar{\eta} \frac{D_{1} u+\partial_{3} u D_{2} u}{1+\left(\partial_{3} u\right)^{2}} \partial_{3} \bar{u}+\frac{\partial_{3} u \partial_{3} f \partial_{3} \bar{u}}{1+\left(\partial_{3} u\right)^{2}}\right) \partial_{3} \bar{u} \\
& +2 \bar{\eta} D_{2} u\left(\frac{D_{2} \partial_{3} \bar{u}-\partial_{3} u D_{1} \partial_{3} \bar{u}}{1+\left(\partial_{3} u\right)^{2}}+\bar{\eta} \frac{D_{2} u-\partial_{3} u D_{1} u}{1+\left(\partial_{3} u\right)^{2}} \partial_{3} \bar{u}+\frac{\partial_{3} f}{1+\left(\partial_{3} u\right)^{2}} \partial_{3} \bar{u}\right) \partial_{3} \bar{u} \\
& \quad+2 \bar{\eta} D_{3} u\left(\frac{D_{3} \partial_{3} \bar{u}}{1+\left(\partial_{3} u\right)^{2}}+\bar{\eta} \frac{D_{3} u}{1+\left(\partial_{3} u\right)^{2}} \partial_{3} \bar{u}\right) \partial_{3} \bar{u}= \\
& =\sum_{j=1}^{3} \beta_{j, 1} D_{j}\left(\left(\partial_{3} \bar{u}\right)^{2}\right)+2 \bar{\eta}^{2} \sum_{j=1}^{3} \frac{\left(D_{j} u\right)^{2}}{1+\left(\partial_{3} u\right)^{2}}\left(\partial_{3} \bar{u}\right)^{2}+ \\
& \quad+\left(2 \bar{\eta} D_{1} u \frac{\partial_{3} u}{1+\left(\partial_{3} u\right)^{2}} \partial_{3} f+2 \bar{\eta} D_{2} u \frac{\partial_{3} f \partial_{3} u}{1+\left(\partial_{3} u\right)^{2}}\right)\left(\partial_{3} \bar{u}\right)^{2}
\end{aligned}
$$

where

$$
\beta_{1,1}=2 \bar{\eta} \frac{D_{1} u-\partial_{3} u D_{2} u}{1+\left(\partial_{3} u\right)^{2}}, \beta_{2,1}=2 \bar{\eta} \frac{D_{1} u \partial_{3} u+D_{2} u}{1+\left(\partial_{3} u\right)^{2}}, \beta_{3,1}=2 \bar{\eta} \frac{D_{3} u}{1+\left(\partial_{3} u\right)^{2}} .
$$

A Hölder type inequality then yields

$$
\begin{aligned}
A_{3} \partial_{3} \bar{u} & \leq \sum_{j=1}^{3} \beta_{j, 1} D_{j}\left(\left(\partial_{3} \bar{u}\right)^{2}\right)+ \\
& +\bar{\eta}^{2} \sum_{j=1}^{3}\left(D_{j} u\right)^{2} \frac{3}{1+\left(\partial_{3} u\right)^{2}}\left(\partial_{3} \bar{u}\right)^{2}+\left(\partial_{3} f\right)^{2}\left(\partial_{3} \bar{u}\right)^{2} .
\end{aligned}
$$

For the terms $A_{4}$ and $A_{5}$ we have

$$
\begin{aligned}
A_{4} & +A_{5}=\partial_{3}\left(\frac{\partial_{1} f+D_{2} u \partial_{3} f}{\psi^{\prime}}\right)-\partial_{3}\left(\partial_{2} f-D_{1} u \partial_{3} f+f \partial_{3} f\right) \partial_{3} \bar{u}= \\
& =-\bar{\eta}\left(\partial_{1} f+D_{2} u \partial_{3} f\right) \partial_{3} \bar{u}+\frac{1}{\psi^{\prime}}\left(\partial_{13} f+D_{2} u \partial_{33} f+\partial_{3} D_{2} u \partial_{3} f\right)- \\
& -\left(\partial_{23} f-D_{1} u \partial_{33} f+f \partial_{33} f-\partial_{3}\left(D_{1} u-f\right) \partial_{3} f\right) \partial_{3} \bar{u}
\end{aligned}
$$

Moreover

$$
\begin{array}{r}
\frac{1}{\psi^{\prime}} \partial_{3} f \partial_{3} D_{2} u-\partial_{3} f \partial_{3}\left(D_{1} u-f\right) \partial_{3} \bar{u}=\frac{1}{\psi^{\prime}} \partial_{3} f\left(\partial_{3} D_{2} u-\partial_{3}\left(D_{1} u-f\right) \partial_{3} u\right)= \\
=\frac{\partial_{3} f}{\psi^{\prime}} D_{2} \partial_{3} u=\partial_{3} f D_{2} \partial_{3} \bar{u}+\partial_{3} f \bar{\eta} D_{2} u \partial_{3} \bar{u}
\end{array}
$$

owing to (1.5) and (2.20). 
Then

$$
\begin{aligned}
A_{4}+A_{5}= & -\bar{\eta} \partial_{1} f \partial_{3} \bar{u}+\frac{1}{\psi^{\prime}}\left(\partial_{13} f+D_{2} u \partial_{33} f\right)- \\
& -\left(\partial_{23} f-D_{1} u \partial_{33} f+f \partial_{33} f\right) \partial_{3} \bar{u}+\partial_{3} f D_{2} \partial_{3} \bar{u}
\end{aligned}
$$

Since

$$
\frac{1}{\psi^{\prime}}\left(\partial_{13} f+D_{2} u \partial_{33} f\right)=\frac{1}{\partial_{3} u}\left(\partial_{13} f+D_{2} u \partial_{33} f\right) \partial_{3} \bar{u}
$$

in the second and third term we obtain:

$$
\begin{aligned}
\left(A_{4}+A_{5}\right) \partial_{3} \bar{u}= & \sum_{j=1}^{3} \beta_{j, 2} D_{j}\left(\left(\partial_{3} \bar{u}\right)^{2}\right)-\bar{\eta} \partial_{1} f\left(\partial_{3} \bar{u}\right)^{2}+ \\
& +\left(\frac{\partial_{13} f}{\partial_{3} u}+\frac{D_{2} u \partial_{33} f}{\partial_{3} u}-\partial_{32} f+D_{1} u \partial_{33} f-f \partial_{33} f\right)\left(\partial_{3} \bar{u}\right)^{2}
\end{aligned}
$$

where we have denoted

$$
\beta_{1,2}=\beta_{3,2}=0, \beta_{2,2}=\frac{\partial_{3} f}{2} .
$$

Thus the last sum is not less than

$$
\begin{aligned}
& \sum_{j=1}^{3} \beta_{j, 2} D_{j}\left(\left(\partial_{3} \bar{u}\right)^{2}\right)-\bar{\eta}^{2} \sum_{j=1}^{3} \frac{\left(D_{j} u\right)^{2}}{2}\left(\partial_{3} \bar{u}\right)^{2}+ \\
+ & \left(-\bar{\eta} \partial_{1} f+\frac{\partial_{13} f}{\partial_{3} u}-\frac{\left|\partial_{33} f\right|^{2}}{\bar{\eta}^{2}}\left(\frac{1}{\left(\partial_{3} u\right)^{2}}+1\right)-\partial_{32} f-f \partial_{33} f\right)\left(\partial_{3} \bar{u}\right)^{2} .
\end{aligned}
$$

Finally set

$$
\left(A_{6}+A_{7}+A_{8}\right) \partial_{3} \bar{u}=\sum_{j=1}^{3} \beta_{j, 3} D_{j}\left(\left(\partial_{3} \bar{u}\right)^{2}\right),
$$

where

$$
\beta_{1,3}=-\partial_{3} a, \beta_{2,3}=-\partial_{3} b, \beta_{3,3}=\partial_{3} u D_{3} v .
$$

Multiplying (2.17) by $\partial_{3} \bar{u}$ and inserting (2.19), (2.21), (2.22), (2.23), for $\left|\partial_{3} \bar{u}\right| \geq \beta$ we finally obtain

$$
\begin{aligned}
& \frac{1}{2} \sum_{j=1}^{3} D_{j}^{2}\left(\left(\partial_{3} \bar{u}\right)^{2}\right)-\sum_{j=1}^{3}\left(D_{j} \partial_{3} \bar{u}\right)^{2}+\sum_{m, j=1}^{3} \beta_{m, j} D_{m}\left(\left(\partial_{3} \bar{u}\right)^{2}\right) \geq \\
\geq & -\frac{\bar{\eta}^{\prime}}{\psi^{\prime}} \sum_{j=1}^{3}\left(D_{j} u\right)^{2}\left(\partial_{3} \bar{u}\right)^{2}+\left(\bar{\eta}^{2} \sum_{j=1}^{3}\left(D_{j} u\right)^{2}\left(1-\frac{3}{1+\left(\partial_{3} u\right)^{2}}-\frac{1}{2}\right)\right)\left(\partial_{3} \bar{u}\right)^{2}+ \\
+ & \left(-\bar{\eta} \partial_{1} f-\frac{\left|\partial_{13} f\right|}{\beta}-\frac{\left|\partial_{33} f\right|^{2}}{\bar{\eta}^{2}}\left(\frac{1}{\beta^{2}}+1\right)-\partial_{32} f-f \partial_{33} f-\left|\partial_{3} f\right|^{2}\right)\left(\partial_{3} \bar{u}\right)^{2} .
\end{aligned}
$$


Now take $\bar{\eta}=\alpha$, where the constants $\alpha$ and $\beta$ are as in (2.13), and accordingly choose $\psi$ such that

$$
\frac{\psi^{\prime \prime}}{\left(\psi^{\prime}\right)^{2}}=\bar{\eta}
$$

With this choice of $\psi$ the right hand side is positive as well as the second term at the left hand side. Consequently,

$$
\frac{1}{2} \sum_{j=1}^{3} D_{j}^{2}\left(\left(\partial_{3} \bar{u}\right)^{2}\right)+\sum_{m, j=1}^{3} \beta_{m, j} D_{m}\left(\left(\partial_{3} \bar{u}\right)^{2}\right) \geq 0 .
$$

In view of the maximum principle, applied to the function $\left|\partial_{3} \bar{u}\right|^{2}$, on the set $\left\{x:\left|\partial_{3} \bar{u}\right|^{2} \geq \beta^{2}\right\}$, we get $\left|\partial_{3} \bar{u}\right| \leq \beta+\max _{b \Omega}|\nabla u|$. This concludes the proof.

Proposition 2.6 Let $u \in C^{3}(\Omega) \cap C^{2}(\bar{\Omega})$ be a solution to (1.9). Then

$$
\left(D_{1} u\right)^{2}+\left(D_{2} u\right)^{2}+\left(D_{3} u\right)^{2} \leq C
$$

where $C$ is a constant depending only on $\max _{b \Omega}|\nabla u|$.

Proof. As in Proposition 2.5 we take an invertibile function $\psi$ such that $u=\psi(\bar{u})$. We also define

$$
\bar{w}^{2}=\sum_{j=1}^{2}\left(D_{j} \bar{u}\right)^{2}+\varepsilon^{2}\left(\partial_{3} \bar{u}\right)^{2} .
$$

Denoting $\eta=\psi^{\prime \prime} / \psi^{\prime}$, from (2.15) and (2.17) we deduce that $\bar{u}$ is a solution of

$$
\sum_{j=1}^{3} D_{j}^{2} \bar{u}+\eta \sum_{j=1}^{3}\left(D_{j} \bar{u}\right)^{2}-\frac{\partial_{1} f}{\psi^{\prime}}-\partial_{3} f D_{2} \bar{u}=0 .
$$

Applying $D_{1}$, in view of $(2.10)$ we obtain

$$
\begin{aligned}
& \sum_{j=1}^{3} D_{j}^{2}\left(D_{1} \bar{u}\right)+2 \frac{D_{3} \partial_{3} u D_{23} \bar{u}}{1+\left(\partial_{3} u\right)^{2}}-2 \frac{D_{2} \partial_{3} u D_{3}^{2} \bar{u}}{1+\left(\partial_{3} u\right)^{2}}+\eta^{\prime} D_{1} \bar{u} \sum_{j=1}^{3}\left(D_{j} \bar{u}\right)^{2} \\
& +2 \eta \sum_{j=1}^{3} D_{j} \bar{u} D_{1 j} \bar{u}-D_{1} \frac{\partial_{1} f}{\psi^{\prime}}-D_{1} \partial_{3} f D_{2} \bar{u}-\partial_{3} f D_{12} \bar{u} \\
& -2 \partial_{3} u \frac{\partial_{3} f}{1+\left(\partial_{3} u\right)^{2}} D_{3}^{2} \bar{u}+\varepsilon \frac{\partial_{33} f}{\left(1+\left(\partial_{3} u\right)^{2}\right)^{3 / 2}} \partial_{3} u D_{3} \bar{u}+ \\
& +2\left(1-\left(\partial_{3} u\right)^{2}\right) \frac{\partial_{3} f}{\left(1+\left(\partial_{3} u\right)^{2}\right)^{2}} D_{3} \partial_{3} u D_{3} \bar{u}=0 .
\end{aligned}
$$


Let us denote $A_{1}, \ldots, A_{11}$ the terms in (2.27). Arguing as in (2.19) we have

$$
D_{1} \bar{u} A_{1}=D_{1} \bar{u} \sum_{j=1}^{3} D_{j}^{2} D_{1} \bar{u}=\frac{1}{2} \sum_{j=1}^{3} D_{j}^{2}\left(\left(D_{1} \bar{u}\right)^{2}\right)-\sum_{j=1}^{3}\left(D_{j} D_{1} \bar{u}\right)^{2} .
$$

In order to treat $A_{2}+A_{3}$ we first observe that, by (2.20)

$$
D_{j} \partial_{3} u=\psi^{\prime} D_{j} \partial_{3} \bar{u}+\psi^{\prime} \bar{\eta} D_{j} u \partial_{3} \bar{u}=\psi^{\prime} D_{j} \partial_{3} \bar{u}+\eta D_{j} u \partial_{3} \bar{u}
$$

and consequently

$$
\begin{aligned}
D_{j} D_{3} \bar{u} & =D_{j}\left(\frac{\varepsilon \partial_{3} \bar{u}}{\left(1+\left(\partial_{3} u\right)^{2}\right)^{1 / 2}}\right)=\frac{\varepsilon D_{j} \partial_{3} \bar{u}}{\left(1+\left(\partial_{3} u\right)^{2}\right)^{1 / 2}}-\frac{\varepsilon \partial_{3} \bar{u} \partial_{3} u D_{j} \partial_{3} u}{\left(1+\left(\partial_{3} u\right)^{2}\right)^{3 / 2}} \\
& =\frac{\varepsilon D_{j} \partial_{3} \bar{u}}{\left(1+\left(\partial_{3} u\right)^{2}\right)^{1 / 2}}-\frac{\varepsilon\left(\partial_{3} u\right)^{2} D_{j} \partial_{3} \bar{u}}{\left(1+\left(\partial_{3} u\right)^{2}\right)^{3 / 2}}-\frac{\varepsilon \partial_{3} \bar{u}\left(\partial_{3} u\right)^{2} \eta D_{j} \bar{u}}{\left(1+\left(\partial_{3} u\right)^{2}\right)^{3 / 2}}= \\
& =\frac{\varepsilon D_{j} \partial_{3} \bar{u}}{\left(1+\left(\partial_{3} u\right)^{2}\right)^{3 / 2}}-\frac{\varepsilon \partial_{3} \bar{u}\left(\partial_{3} u\right)^{2} \eta D_{j} \bar{u}}{\left(1+\left(\partial_{3} u\right)^{2}\right)^{3 / 2}} .
\end{aligned}
$$

Then

$$
\begin{aligned}
& A_{2}+A_{3}=2 \frac{\psi^{\prime} D_{3} \partial_{3} \bar{u}+\eta D_{3} \bar{u} \partial_{3} u}{\left(1+\left(\partial_{3} u\right)^{2}\right)^{5 / 2}}\left(\varepsilon D_{2} \partial_{3} \bar{u}-\varepsilon \eta\left(\partial_{3} u\right)^{2} D_{2} \bar{u} \partial_{3} \bar{u}\right)- \\
& -2 \frac{\psi^{\prime} D_{2} \partial_{3} \bar{u}+\eta D_{2} \bar{u} \partial_{3} u}{\left(1+\left(\partial_{3} u\right)^{2}\right)^{5 / 2}}\left(\varepsilon D_{3} \partial_{3} \bar{u}-\varepsilon \eta\left(\partial_{3} u\right)^{2} D_{3} \bar{u} \partial_{3} \bar{u}\right)= \\
= & \frac{2 \eta D_{2}\left(\varepsilon \partial_{3} \bar{u}\right) \partial_{3} u D_{3} \bar{u}}{\left(1+\left(\partial_{3} u\right)^{2}\right)^{5 / 2}}-\frac{2 \eta D_{3}\left(\varepsilon \partial_{3} \bar{u}\right)\left(\partial_{3} u\right)^{3} D_{2} \bar{u}}{\left(1+\left(\partial_{3} u\right)^{2}\right)^{5 / 2}}-\frac{2 \eta D_{3}\left(\varepsilon \partial_{3} \bar{u}\right) \partial_{3} u D_{2} \bar{u}}{\left(1+\left(\partial_{3} u\right)^{2}\right)^{5 / 2}} \\
& +\frac{2 \eta D_{2}\left(\varepsilon \partial_{3} \bar{u}\right)\left(\partial_{3} u\right)^{3} D_{3} \bar{u}}{\left(1+\left(\partial_{3} u\right)^{2}\right)^{5 / 2}}=\frac{2 \eta D_{2}\left(\varepsilon \partial_{3} \bar{u}\right) \partial_{3} u D_{3} \bar{u}}{\left(1+\left(\partial_{3} u\right)^{2}\right)^{3 / 2}}-\frac{2 \eta D_{3}\left(\varepsilon \partial_{3} \bar{u}\right) \partial_{3} u D_{2} \bar{u}}{\left(1+\left(\partial_{3} u\right)^{2}\right)^{3 / 2}}
\end{aligned}
$$

and

$$
\left|D_{1} \bar{u}\right|\left|A_{2}+A_{3}\right| \leq \delta \sum_{j=2}^{3}\left(D_{j}\left(\varepsilon \partial_{3} \bar{u}\right)\right)^{2}+\frac{1}{\delta} \eta^{2} \bar{w}^{4}
$$

where $\bar{w}$ is defined in (2.25) and $\delta$ is a suitable constant to be chosen later.

Similarly,

$$
\begin{aligned}
& \left|D_{1} \bar{u} A_{5}\right|=\left|D_{1} \bar{u}\right|\left|2 \eta \sum_{j=1}^{2} D_{j} \bar{u} D_{1} D_{j} \bar{u}+2 \eta D_{3} \bar{u} D_{1} D_{3} \bar{u}\right| \leq \\
& \leq\left|D_{1} \bar{u}\right|\left|2 \eta \sum_{j=1}^{2} D_{j} \bar{u} D_{1} D_{j} \bar{u}\right|+\left|D_{1} \bar{u}\right|\left|\frac{2 \eta \varepsilon \partial_{3} \bar{u} D_{1}\left(\varepsilon \partial_{3} \bar{u}\right)}{\left(1+\left(\partial_{3} u\right)^{2}\right)^{2}}\right|+ \\
& +\left|D_{1} \bar{u}\right|\left|\frac{2 \eta^{2}\left(\varepsilon \partial_{3} \bar{u}\right)^{3}\left(\partial_{3} u\right)^{2}}{\left(1+\left(\partial_{3} u\right)^{2}\right)^{5 / 2}}\right| \leq \frac{3 \eta^{2}}{\delta} w^{4}+\delta \sum_{j=1}^{2}\left|D_{1} D_{j} \bar{u}\right|^{2}+\delta\left|D_{1}\left(\varepsilon \partial_{3} \bar{u}\right)\right|^{2} .
\end{aligned}
$$


For the terms $A_{6}, A_{7}, A_{8}, A_{10}$ we have

$$
\begin{aligned}
& \left|D_{1} \bar{u}\right|\left|A_{6}+A_{7}+A_{8}+A_{10}\right|= \\
= & \mid-\frac{\partial_{11} f+a \partial_{12} f}{\psi^{\prime}}+\partial_{1} f \frac{\eta}{\psi^{\prime}} D_{1} \bar{u}-\left(\partial_{13} f+a \partial_{23} f\right) D_{2} \bar{u}- \\
& -\partial_{3} f D_{1} D_{2} \bar{u}+\varepsilon \frac{\partial_{33} f}{\left(1+\left(\partial_{3} u\right)^{2}\right)^{3 / 2}} \partial_{3} u D_{3} \bar{u}|| D_{1} \bar{u} \mid \leq \\
\leq & \frac{C}{\left|\psi^{\prime}\right|}\left(1+\left|D_{2} \bar{u}\right|\left|\psi^{\prime}\right|+|\eta|\left|D_{1} \bar{u}\right|\right)\left|D_{1} \bar{u}\right|+ \\
& +C\left(1+\left|D_{2} \bar{u}\right|\left|\psi^{\prime}\right|\right)\left|D_{2} \bar{u}\right|\left|D_{1} \bar{u}\right|+\delta\left|D_{1} D_{2} \bar{u}\right|^{2}+\frac{1}{\delta}\left|D_{1} \bar{u}\right|^{2}+\frac{C}{\left|\psi^{\prime}\right|}\left|D_{1} \bar{u}\right| \\
\leq & C\left|\psi^{\prime}\right| \bar{w}^{3}+C\left(1+\frac{1}{\delta}+\frac{|\eta|}{\left|\psi^{\prime}\right|}\right) \bar{w}^{2}+\frac{C}{\left|\psi^{\prime}\right|} \bar{w}+\delta \sum_{j=1}^{2}\left(D_{1 j} \bar{u}\right)^{2},
\end{aligned}
$$

since $a=D_{2} u=\psi^{\prime} D_{2} \bar{u}$ and the derivatives of $f$ are bounded.

For the remaining terms, using (2.29), we have

$$
A_{9}+A_{11}=-2 \partial_{3} u \frac{\partial_{3} f}{1+\left(\partial_{3} u\right)^{2}} D_{3}^{2} \bar{u}+2 \frac{\partial_{3} f\left(1-\left(\partial_{3} u\right)^{2}\right)}{\left(1+\left(\partial_{3} u\right)^{2}\right)^{2}} D_{3} \partial_{3} u D_{3} \bar{u}=
$$$$
=-2 \frac{\partial_{3} u \partial_{3} f}{\left(1+\left(\partial_{3} u\right)^{2}\right)^{5 / 2}} D_{3}\left(\varepsilon \partial_{3} \bar{u}\right)+2 \eta \frac{\left(\partial_{3} u\right)^{3} \partial_{3} f}{\left(1+\left(\partial_{3} u\right)^{2}\right)^{2}}\left(\varepsilon \partial_{3} \bar{u}\right)^{2}+
$$$$
+2 \partial_{3} u\left(1-\left(\partial_{3} u\right)^{2}\right) \frac{\partial_{3} f}{\left(1+\left(\partial_{3} u\right)^{2}\right)^{5 / 2}} D_{3}\left(\varepsilon \partial_{3} \bar{u}\right)+2 \eta \frac{\partial_{3} f \partial_{3} u\left(1-\left(\partial_{3} u\right)^{2}\right)}{\left(1+\left(\partial_{3} u\right)^{2}\right)^{3}}\left(\varepsilon \partial_{3} \bar{u}\right)^{2} .
$$

Then there exists a constant $C$ such that

$$
\left|D_{1} \bar{u}\right|\left|A_{9}+A_{12}\right| \leq \delta\left(D_{3}\left(\varepsilon \partial_{3} \bar{u}\right)\right)^{2}+\frac{C}{\delta}+C|\eta| \bar{w}^{2},
$$

(for a suitable $\delta$ to be chosen later).

Substituting all the above estimates in (2.27) we obtain the following inequality:

$$
\begin{aligned}
& \frac{1}{2} \sum_{j=1}^{3} D_{j}^{2}\left(D_{1} \bar{u}\right)^{2}-\sum_{j=1}^{3}\left(D_{j 1}^{2} \bar{u}\right)^{2} \geq \\
& \geq-3 \delta \sum_{j=1}^{2}\left(D_{1 j}^{2} \bar{u}\right)^{2}-3 \delta \sum_{j=1}^{3}\left(D_{j}\left(\varepsilon \partial_{3} \bar{u}\right)\right)^{2}+\eta^{\prime}\left(D_{1} \bar{u}\right)^{2} \bar{w}^{2}-C \frac{\eta^{2}}{\delta} \bar{w}^{4}- \\
& \quad-\left|\psi^{\prime}\right| \bar{w}^{3}-C\left(1+\frac{1}{\delta}+\frac{|\eta|}{\left|\psi^{\prime}\right|}+|\eta|\right) \bar{w}^{2}-\frac{C}{\left|\psi^{\prime}\right|} \bar{w}-\frac{C}{\delta}
\end{aligned}
$$


We now differentiate (2.26) with respect to $D_{2}$. Owing to (2.11) we obtain

$$
\begin{aligned}
& \sum_{j=1}^{3} D_{j}^{2}\left(D_{2} \bar{u}\right)-2 \frac{D_{3} \partial_{3} u D_{13} \bar{u}}{1+\left(\partial_{3} u\right)^{2}}+2 \frac{D_{1} \partial_{3} u D_{3}^{2} \bar{u}}{1+\left(\partial_{3} u\right)^{2}}+\eta^{\prime} D_{2} \bar{u} \sum_{j=1}^{3}\left(D_{j} \bar{u}\right)^{2}+ \\
& +2 \eta \sum_{j=1}^{3} D_{j} \bar{u} D_{2} D_{j} \bar{u}-D_{2} \frac{\partial_{1} f}{\psi^{\prime}}-D_{2} \partial_{3} f D_{2} \bar{u}-\partial_{3} f D_{2}^{2} \bar{u}- \\
& -2 \frac{\partial_{3} f}{1+\left(\partial_{3} u\right)^{2}} D_{3}^{2} \bar{u}-2 \partial_{3} u \frac{\partial_{3} f}{\left(1+\left(\partial_{3} u\right)^{2}\right)^{2}} D_{3} \partial_{3} u D_{3} \bar{u}=0 .
\end{aligned}
$$

Let us denote $\widetilde{A}_{1}, \ldots, \widetilde{A}_{10}$ the terms in (2.35). We proceed exactly in the same way as for the corresponding terms in (2.27). Here we make explicit some computations just for the reader's convenience.

We have:

$$
\begin{aligned}
D_{2} \bar{u} \widetilde{A}_{1} & =\frac{1}{2} \sum_{j=1}^{3} D_{j}^{2}\left(\left(D_{2} \bar{u}\right)^{2}\right)-\sum_{j=1}^{3}\left(D_{j} D_{2} \bar{u}\right)^{2} \\
\left|D_{2} \bar{u} \| \widetilde{A}_{2}+\widetilde{A}_{3}\right| & =\left|\frac{2 \eta D_{1}\left(\varepsilon \partial_{3} \bar{u}\right) \partial_{3} u D_{3} \bar{u}}{\left(1+\left(\partial_{3} u\right)^{2}\right)^{3 / 2}}-\frac{2 \eta D_{3}\left(\varepsilon \partial_{3} \bar{u}\right) \partial_{3} u D_{1} \bar{u}}{\left(1+\left(\partial_{3} u\right)^{2}\right)^{3 / 2}}\right|\left|D_{2} \bar{u}\right| \leq \\
& \leq \delta \sum_{j=1}^{3}\left(D_{j}\left(\varepsilon \partial_{3} \bar{u}\right)\right)^{2}+\frac{C}{\delta} \eta^{2} \bar{w}^{4}
\end{aligned}
$$

Moreover

$$
\begin{aligned}
& D_{2} \bar{u} \widetilde{A}_{4}=\eta^{\prime}\left(D_{2} \bar{u}\right)^{2}|D \bar{u}|^{2}, \\
& \left|D_{2} \bar{u}\right|\left|\widetilde{A}_{6}+\widetilde{A}_{7}+\widetilde{A}_{8}+\widetilde{A}_{10}\right| \leq\left|D_{2} \bar{u}\right|\left|\frac{\partial_{12} f+b \partial_{12} f}{\psi^{\prime}}-\partial_{1} f \frac{\eta}{\psi^{\prime}} D_{2} \bar{u}\right|+ \\
& \quad+\left|D_{2} \bar{u}\right|\left|\left(\partial_{23} f+b \partial_{33} f\right) D_{2} \bar{u}+\partial_{2} f D_{22} \bar{u}+\varepsilon \frac{\partial_{33} f}{\left(1+\left(\partial_{3} u\right)^{2}\right)^{3 / 2}} \partial_{3} u D_{3} \bar{u}\right| \leq \\
& \quad \leq C\left|\psi^{\prime}\right| \bar{w}^{3}+C\left(1+\frac{1}{\delta}+\frac{|\eta|}{\left|\psi^{\prime}\right|}\right) \bar{w}^{2}+\frac{C}{\left|\psi^{\prime}\right|} \bar{w}+\delta\left(D_{22} \bar{u}\right)
\end{aligned}
$$

and

$$
\left|D_{2} \bar{u}\right|\left|\widetilde{A}_{9}+\widetilde{A}_{11}\right| \leq \delta \sum_{j=1}^{3}\left(D_{3}\left(\varepsilon \partial_{3} \bar{u}\right)\right)^{2}+\frac{C}{\delta}+C|\eta| \bar{w}^{2}
$$


Collecting together all these inequalities we obtain

$$
\begin{aligned}
& \frac{1}{2} \sum_{j=1}^{3} D_{j}^{2}\left(\left(D_{2} \bar{u}\right)^{2}\right)-\sum_{j=1}^{3}\left(D_{j} D_{2} \bar{u}\right)^{2} \geq \\
\geq & -3 \delta \sum_{j=1}^{2}\left(D_{2 j}^{2} \bar{u}\right)^{2}-3 \delta \sum_{j=1}^{2}\left(D_{j}\left(\varepsilon \partial_{3} \bar{u}\right)\right)^{2}+\eta^{\prime}\left(D_{2} \bar{u}\right)^{2} \bar{w}^{2}-\frac{\eta^{2}}{\delta} \bar{w}^{4}- \\
& -C\left|\psi^{\prime}\right| \bar{w}^{3}+C\left(1+\frac{1}{\delta}+\frac{|\eta|}{\left|\psi^{\prime}\right|}+|\eta|\right) \bar{w}^{2}-C \frac{1}{\left|\psi^{\prime}\right|} \bar{w}-\frac{C}{\delta}
\end{aligned}
$$

Finally to treat $\varepsilon \partial_{3} \bar{u}$ we slightly modify the computations in Proposition 2.5. We have already proved that

$$
\begin{aligned}
& \sum_{j=1}^{3} D_{j}^{2}\left(\varepsilon \partial_{3} \bar{u}\right)^{2}-\sum_{j=1}^{3} D_{j}\left(\varepsilon \partial_{3} \bar{u}\right)^{2} \geq-\sum_{m, j=1}^{3} \beta_{m, j} D_{m}\left(\varepsilon \partial_{3} \bar{u}\right)^{2}-\frac{\bar{\eta}^{\prime}}{\psi^{\prime}} \sum_{j=1}^{3}\left(D_{j} u\right)^{2}\left(\varepsilon \partial_{3} \bar{u}\right)^{2} \\
& +\left(\bar{\eta}^{2} \sum_{j=1}^{3}\left(D_{j} u\right)^{2}\left(1-\frac{3}{1+\left(\partial_{3} u\right)^{2}}-\frac{1}{2}\right)-|\bar{\eta}| \sup \left|\partial_{1} f\right|-C\right)\left(\varepsilon \partial_{3} \bar{u}\right)^{2} \geq \\
& \geq-\sum_{m, j=1}^{3} \beta_{m, j} D_{m}\left(\varepsilon \partial_{3} \bar{u}\right)^{2}-\eta^{\prime}(D \bar{u})^{2}\left(\varepsilon \partial_{3} \bar{u}\right)^{2}-C \eta^{2} \bar{w}^{4}-C\left(\left|\eta \psi^{\prime}\right|+1\right) w^{2}
\end{aligned}
$$

since

$$
\left(-\bar{\eta}^{\prime} / \psi^{\prime}+\bar{\eta}^{2}\right)(D u)^{2}=-\frac{\eta^{\prime}}{\left(\psi^{\prime}\right)^{2}}(D u)^{2}=-\eta^{\prime}(D \bar{u})^{2},
$$

and $\bar{\eta} D u=\eta D \bar{u}$.

Let us estimate terms involving $\beta_{m, j}$. We have

$$
\begin{aligned}
\sum_{m=1}^{3} \sum_{j=1}^{2} \beta_{m, j} D_{j}\left(\varepsilon \partial_{3} \bar{u}\right)^{2} & \leq\left(2|\eta|\left|D_{1} u\right|+C\right)\left|\varepsilon \partial_{3} \bar{u}\right|\left|\sum_{j=1}^{2} D_{j}\left(\varepsilon \partial_{3} \bar{u}\right)\right| \leq \\
& \leq \delta \sum_{j=1}^{2}\left(D_{i}\left(\varepsilon \partial_{3} \bar{u}\right)^{2}+\frac{\eta^{2}}{\delta} \bar{w}^{2}+\frac{C}{\delta} .\right.
\end{aligned}
$$

On the other hand, owing to Lemma 2.2 and remembering that $v=$ $\arctan \left(\partial_{3} u\right)$, we have

$$
\begin{aligned}
& -\varepsilon \partial_{3} \bar{u} \sum_{j=1}^{3} \beta_{j 3} D_{j}\left(\varepsilon \partial_{3} \bar{u}\right)= \\
& \quad=-2 \varepsilon \partial_{3} \bar{u}\left(\partial_{3} a D_{1}\left(\varepsilon \partial_{3} \bar{u}\right)+\partial_{3} b D_{2}\left(\varepsilon \partial_{3} \bar{u}\right)-\partial_{3} u D_{3} v D_{3}\left(\varepsilon \partial_{3} \bar{u}\right)\right)=
\end{aligned}
$$




$$
\begin{aligned}
= & -2 \varepsilon \partial_{3} \bar{u} D_{1}\left(\varepsilon \partial_{3} \bar{u}\right)\left(\frac{D_{2} \partial_{3} u-\partial_{3} u D_{1} \partial_{3} u}{1+\left(\partial_{3} u\right)^{2}}+\frac{\partial_{3} u \partial_{3} f}{1+\left(\partial_{3} u\right)^{2}}\right) \\
& -2 \varepsilon \partial_{3} \bar{u} D_{2}\left(\varepsilon \partial_{3} \bar{u}\right)\left(-\frac{D_{1} \partial_{3} u+\partial_{3} u D_{2} \partial_{3} u}{1+\left(\partial_{3} u\right)^{2}}+\frac{\partial_{3} f}{1+\left(\partial_{3} u\right)^{2}}\right) \\
& +2 \varepsilon \partial_{3} \bar{u} \frac{\partial_{3} u D_{3} \partial_{3} u D_{3}\left(\varepsilon \partial_{3} \bar{u}\right)}{1+\left(\partial_{3} u\right)^{2}}
\end{aligned}
$$

(by $(2.28)$ )

$$
\begin{gathered}
=-2 \varepsilon \partial_{3} u D_{1}\left(\varepsilon \partial_{3} \bar{u}\right)\left(\frac{D_{2} \partial_{3} \bar{u}-\partial_{3} u D_{1} \partial_{3} \bar{u}}{1+\left(\partial_{3} u\right)^{2}}+\eta \frac{D_{2} \bar{u}-\partial_{3} u D_{1} \bar{u}}{1+\left(\partial_{3} u\right)^{2}} \partial_{3} \bar{u}+\frac{\partial_{3} \bar{u} \partial_{3} f}{1+\left(\partial_{3} u\right)^{2}}\right) \\
-2 \varepsilon \partial_{3} u D_{2}\left(\varepsilon \partial_{3} \bar{u}\right)\left(-\frac{D_{1} \partial_{3} \bar{u}+\partial_{3} u D_{2} \partial_{3} \bar{u}}{1+\left(\partial_{3} u\right)^{2}}-\eta \partial_{3} \overline{D_{1}} \frac{D_{1} \bar{u}+\partial_{3} u D_{2} \bar{u}}{1+\left(\partial_{3} u\right)^{2}}+\right. \\
\left.+\frac{\partial_{3} f}{\left(1+\left(\partial_{3} u\right)^{2}\right) \psi^{\prime}}\right)+2 \varepsilon \partial_{3} u \frac{\partial_{3} u D_{3}\left(\varepsilon \partial_{3} \bar{u}\right)}{1+\left(\partial_{3} u\right)^{2}}\left(D_{3}\left(\partial_{3} \bar{u}\right)+\eta D_{3} \bar{u} \partial_{3} \bar{u}\right) \\
\geq 2 \varepsilon^{2}\left(\partial_{3} u\right)^{2} \frac{\left(D_{1} \partial_{3} \bar{u}\right)^{2}+\left(D_{2} \partial_{3} \bar{u}\right)^{2}+\left(D_{3} \partial_{3} \bar{u}\right)^{2}}{1+\left(\partial_{3} u\right)^{2}}-\delta \sum_{j=1}^{3}\left(D_{j}\left(\varepsilon \partial_{3} \bar{u}\right)\right)^{2}- \\
-C \frac{\eta^{2}}{\delta} \bar{w}^{4}-C \bar{w}^{2}-\frac{C}{\psi^{\prime 2}} .
\end{gathered}
$$

Hence

$$
\begin{gathered}
\sum_{j=1}^{3} D_{j}^{2}\left(\varepsilon \partial_{3} \bar{u}\right)^{2}-\sum_{j=1}^{3} D_{j}\left(\varepsilon \partial_{3} \bar{u}\right)^{2} \geq-\delta \sum_{j=1}^{3}\left(D_{j}\left(\varepsilon \partial_{3} \bar{u}\right)\right)^{2}-\eta^{\prime} \bar{w}^{2}\left(\varepsilon \partial_{3} \bar{u}\right)^{2} \\
-C \eta^{2}\left(1+\frac{1}{\delta}\right) \bar{w}^{4}-C\left(|\eta|\left|\psi^{\prime}\right|+1+\frac{\eta^{2}}{\delta}\right) \bar{w}^{2}-\frac{C}{\psi^{\prime 2}}-\frac{C}{\delta}
\end{gathered}
$$

Summing (2.34), (2.36), (2.37), we finally obtain

$$
\begin{aligned}
& \frac{1}{2} \sum_{i=1}^{3} D_{i}^{2} \bar{w}^{2} \geq(1-6 \delta) \sum_{i=1}^{3} \sum_{j=1}^{2}\left(D_{i} D_{j} \bar{u}\right)^{2}-(1-7 \delta) \sum_{i=1}^{3} D_{i}\left(\varepsilon \partial_{3} \bar{u}\right)^{2}- \\
& \quad-\left(\eta^{\prime}-C \eta^{2}\left(1+\frac{1}{\delta}\right)\right) \bar{w}^{4}-C\left|\psi^{\prime}\right| \bar{w}^{3}- \\
& \quad-C\left(1+\frac{1}{\delta}+\frac{|\eta|}{\left|\psi^{\prime}\right|}+|\eta|\left|\psi^{\prime}\right|+\frac{\eta^{2}}{\delta}\right) \bar{w}^{2}-C\left(1+\frac{1}{\left|\psi^{\prime}\right|}\right) \bar{w}-\frac{C}{\psi^{\prime 2}}-\frac{C}{\delta} .
\end{aligned}
$$

Now fix $\delta \leq 1 / 8$ so that the first two terms of the second member are positive. Then, reminding that $\eta=\psi^{\prime \prime} / \psi^{\prime}$, make a choice of $\psi$ so as to have

$$
-\eta^{\prime}>C \eta^{2}\left(1+\frac{1}{\delta}\right)=C_{1} \eta^{2}
$$

$C_{1}$ being a fixed constant. 
For instance the function $\psi:[\tau, 2 \tau] \rightarrow \mathbb{R}$ defined by

$$
\psi(s)=C_{2} \int_{\tau}^{s} \exp \left(-\sigma^{2}\right) d \sigma+m
$$

where $m=\min u$, and $C_{2}$ is such that $\psi(2 \tau)>\max u$, has the required property. With this choice of $\psi$, we have

$$
\eta=-4 s, \quad \eta^{\prime}=-4
$$

and, consequently,

$$
-\eta^{\prime}-C_{1} \eta^{2} \geq 4-\frac{16 \tau^{2}}{\delta} \geq 2,
$$

for a suitable $\tau$. It follows

$$
\begin{aligned}
\frac{1}{2} \sum_{i=1}^{3} D_{i}^{2} \bar{w}^{2} \geq & 2 \bar{w}^{4}-C\left|\psi^{\prime}\right| \bar{w}^{3}-C\left(1+\frac{1}{\delta}+\frac{|\eta|}{\left|\psi^{\prime}\right|}+\left|\eta \psi^{\prime}\right|+\frac{\eta^{2}}{\delta}\right) \bar{w}^{2} \\
& +C\left(1+\frac{1}{\left|\psi^{\prime}\right|}\right) \bar{w}-\frac{C}{\psi^{\prime 2}}-\frac{C}{\delta}
\end{aligned}
$$

and the right hand side is positive if $\bar{w} \gg 0$. Thus, if $\bar{w}^{2} \gg 0, \bar{w}^{2}$ is a subsolution of an elliptic operator and then we invoke the maximum principle to conclude the proof.

\subsection{Existence of a solution}

Let us state the existence theorem for the Dirichlet problem (1.9):

Theorem 2.7 Let $f \in C^{k, \alpha}(\bar{\Omega}), k \geq 2$, satisfy the hypothesis of Proposition 2.5. Assume that $b \Omega$ is strictly $J$-pseudoconvex, and that the boundary value $g$ is of class $C^{2, \alpha}$. Then there exists a unique solution $u \in C^{k+1, \alpha}(\Omega) \cap$ $C^{2, \alpha}(\bar{\Omega})$ of problem (1.9). Moreover

$$
\left(D_{1} u\right)^{2}+\left(D_{2} u\right)^{2}+\left(D_{3} u\right)^{2}+\left(\partial_{3} u\right)^{2} \leq K
$$

where $K$ is a constant depending only on $u_{\mid b \Omega}$ and $\nabla u_{\mid b \Omega}$.

Proof. The existence of the solution follows, by standard procedure, from (2.3) in Section 2.1 and Proposition 2.6. The uniqueness is a consequence of the comparison principle stated in Section 3.1.

Remark 2.1 Note that the hypothesis of Proposition 2.5 is satisfied if either $f=0$ or $\sup \partial_{1} f<0$. 


\section{A priori estimates in the Sobolev spaces}

In this section we introduce some Sobolev spaces naturally defined in terms of the vector fields $D_{j}$. Then, suitably adapting a technique introduced in $[\mathrm{CM} 1],[\mathrm{CLM}],[\mathrm{CM} 2]$ for the standard complex structure $(f=0)$ we prove some uniform (with respect to $\varepsilon$ ) estimates of the solution of the approximated problem (1.9). For this purpose we study the linear equation

$$
\sum_{j=1}^{3} D_{j}^{2} w=v_{0}+\sum_{j=1}^{3} v_{j} D_{j} w
$$

which has the same structure as the (elliptic regularization of the) Levi equation. Here the coefficients of the vector fields $D_{1}=\partial_{1}+a \partial_{3}, D_{2}=$ $\partial_{2}+b \partial_{3}$ depend upon a fixed function $u: a=a(u), b=b(u)$. Although some of the statements below are valid for a general $u$ we assume that $u$ is a solution of the approximated problem.

Let us recall the definition of weak derivative. We say that a function $w \in$ $L_{\mathrm{loc}}^{1}(\Omega)$ is weakly differentiable with respect to $D_{j}$ if there exists a function $v \in L_{\mathrm{loc}}^{1}(\Omega)$ such that

$$
\int w D_{j}^{*} \phi d \lambda=\int v \phi d \lambda \quad \forall \phi \in C_{0}^{\infty}(\Omega)
$$

where $D_{j}^{*}$ is the formal adjoint of $D_{j}$ (and $d \lambda$ the Lebesgue measure).

For any domain $U \subset \Omega$ let

$$
\begin{gathered}
W_{\varepsilon}^{m, p}(U)=\left\{w \in L_{\mathrm{loc}}^{1}(U): D_{I} w \in L^{p}(U), \forall I:|I| \leq m\right\} \\
\|w\|_{W_{\varepsilon}^{m, p}(U)}=\sum_{|I| \leq m}\left\|D_{I} w\right\|_{L^{p}(U)} .
\end{gathered}
$$

In particular

$$
\|w\|_{W_{\varepsilon}^{0, p}(U)}=\|w\|_{L^{p}(U)} .
$$

A function $w \in L_{\text {loc }}^{1}(\Omega)$ is said to be in $W_{\varepsilon, \text { loc }}^{m, p}(\Omega)$ if $w \in W_{\varepsilon}^{m, p}(U)$ for every domain $U \Subset \Omega$. Finally $W^{m, p}(\Omega)=W_{1}^{m, p}(\Omega)$ is the usual Sobolev space.

From now on we denote by $K$ a fixed constant as in the estimate (2.38). In the sequel we need the following simple lemma:

Lemma 3.1 Let $u \in W_{\varepsilon}^{m, m p}(\Omega)$ and $v=\arctan \left(\partial_{3} u\right)$. Assume that $v \in$ $W_{\varepsilon}^{m, m p}(\Omega)$ and that $f \in C^{m+1}(\Omega)$. Then there exists a constant $C=$ $C(\Omega, K)$ such that

$$
\begin{aligned}
& \left\|F\left(\partial_{3} u\right) \partial_{3} f\right\|_{W^{m, p}(\Omega)}^{p} \leq \\
& \quad \leq C\left\|\nabla^{m+1} f\right\|_{\infty} \sup _{s \in[-K, K], m \leq|I|}\left|\nabla^{m} F(s)\right|\left(\|v\|_{W_{\varepsilon}^{m, m p}(\Omega)}^{m p}+\|u\|_{W_{\varepsilon}^{m, m p}(\Omega)}^{m p}\right)
\end{aligned}
$$

for any function $F \in C^{\infty}(\mathbb{R})$. 
Proof. Denote $\nabla_{\sigma}=\partial_{\sigma_{1}} \cdots \partial_{\sigma_{k}}, \sigma=\left(\sigma_{1}, \ldots, \sigma_{k}\right)$. Since $a=D_{2} u$,

$$
D_{1} \partial_{3} f=\partial_{13} f+D_{2} u \partial_{33} f \text {. }
$$

Let $I$ be a multi-index. By a simple induction argument it is shown that there are integers $T_{I \sigma}$, and a family $\mathbf{I}_{\sigma, t, I}$ of multi-indices, $|\sigma| \leq|I|, l \leq T_{I \sigma}$, such that:

$$
D_{I} \partial_{3} f=\sum_{|\sigma| \leq|I|} c_{\sigma} \sum_{l=1}^{T_{I \sigma}}\left(\prod_{\rho \in \mathbf{I}_{\sigma, l, I}} D_{\rho} u\right) \nabla_{\sigma} \partial_{3} f,
$$

where $c_{\sigma}$ 's are suitable constants, (possibly zero) and for every $\sigma, \mathbf{I}_{\sigma, t, I}$ contains at most $|I|$ multi-indices of length less or equal $|I|$. Assume that this is true for any multi-index of length $s$. Let $I$ be of length $l=s+1$. Then $I=\left(j, I^{\prime}\right)$, with $\left|I^{\prime}\right|=s$. For simplicity we assume $j=1$. Since $D_{1}=\partial_{1}+D_{2} u \partial_{3}$, differentiating the previous expression we have:

$$
\begin{gathered}
D_{I} \partial_{3} f=D_{1} D_{I^{\prime}}\left(\partial_{3} f\right)=\sum_{|\sigma| \leq s} C_{\sigma} \sum_{l=1}^{T_{I \sigma}} D_{1}\left(\prod_{\rho \in \mathbf{I}_{\sigma, l, I}} D_{\rho} u\right) \nabla_{\sigma} \partial_{3} f+ \\
+\sum_{|\sigma| \leq s} C_{\sigma} \sum_{l=1}^{T_{I \sigma}}\left(\prod_{\rho \in \mathbf{I}_{\sigma, l, I}} D_{\rho} u\right) \partial_{1} \nabla_{\sigma} \partial_{3} f+\sum_{|\sigma| \leq s} C_{\sigma} \sum_{l=1}^{T_{I \sigma}}\left(\prod_{\rho \in \mathbf{I}_{\sigma, l, I}} D_{\rho} u\right) D_{2} u \partial_{3} \nabla_{\sigma} \partial_{3} f .
\end{gathered}
$$

Note that

$$
\sum_{l=1}^{T_{I \sigma}} D_{1}\left(\prod_{\rho \in \mathbf{I}_{\sigma, l, I}} D_{\rho} u\right)=\sum_{l=1}^{\widetilde{T}_{I \sigma}} \prod_{\rho \in I_{\sigma, l, I}} D_{\rho} u
$$

where $\widetilde{\mathbf{I}}_{\sigma, l, I}$ contains at most $s$ multi-indices of length less or equal $s+1$, while

$$
\sum_{l=1}^{T_{I \sigma}}\left(\prod_{\rho \in \mathbf{I}_{\sigma, l, I}} D_{\rho} u\right) D_{2} u=\sum_{t=1}^{\hat{T}_{I \sigma}} \prod_{\rho \in \widetilde{\mathbf{I}}_{\sigma, t, I}} D_{\rho} u
$$

where $\widetilde{\mathbf{I}}_{\sigma, l, I}$ contains at most $s+1$ multi-indices of length $l \leq s$. Relation (3.3) is then proved. By the chain rule

$$
D_{I}\left(F\left(\partial_{3} u\right)\right)=\sum_{l=1}^{|I|} F^{(l)}\left(\partial_{3} u\right) \sum_{I=\left(\sigma_{1}, \cdots, \sigma_{l}\right)}\left(\prod_{j=1}^{t} D_{\sigma_{j}} \partial_{3} u\right)
$$

where $F^{(t)}$ is the $l^{\text {th }}$ derivative of $F$. Then, if $L_{I}$ denotes the set of ordered submulti-indices of $I$,

$$
D_{I}\left(F\left(\partial_{3} u\right) \partial_{3} f\right)=\sum_{\tau \in L_{I}} D_{I-\tau} F\left(\partial_{3} u\right) D_{\tau} \partial_{3} f=
$$




$$
=\sum_{\tau \in L_{I}} \sum_{l=1}^{|I|-|\tau|} F^{(l)}\left(\partial_{3} u\right) \sum_{I-\tau=\left(\sigma_{1}, \cdots, \sigma_{l}\right)}\left(\prod_{j=1}^{l} D_{\sigma_{j}} \partial_{3} u\right) \sum_{|\sigma| \leq|\tau|} c_{\sigma} \sum_{l=1}^{T_{I \sigma}}\left(\prod_{\rho \in I_{\sigma \tau}} D_{\rho} u\right) \nabla_{\sigma} \partial_{3} f .
$$

We obtain the assertion taking the $L^{p}$ norms.

The main property of the solutions of equation (3.1) is expressed by the following:

Theorem 3.2 Let $p \geq 3, m \in \mathbb{N} \backslash\{0\}$ be fixed. Assume that $f \in C^{m+1}(\Omega)$, $u, v=\arctan \left(\partial_{3} u\right) \in W_{\varepsilon, \text { loc }}^{m, m p}(\Omega)$. Also assume that $v_{0} \in W_{\varepsilon, \text { loc }}^{m, 2 p}(\Omega), v_{i} \in$ $W_{\varepsilon, \text { loc }}^{m, p}(\Omega) \cap W_{\varepsilon, \text { loc }}^{m-1,2 p}(\Omega)$, and let $w$ be a solution of equation (3.1). If $\Omega_{1} \Subset$ $\Omega_{2} \Subset \Omega$ then there exists a constant $C=C\left(p, f, \Omega_{1}, \Omega_{2}, K\right)$, such that the following estimate holds true

$$
\begin{aligned}
\|w\|_{W_{\varepsilon}^{m+1, p}\left(\Omega_{1}\right)}^{p} & +\sum_{|I|=m+1}\left\|\left|D_{i} w\right|^{(p-1) / 2}\right\|_{W_{\varepsilon}^{1,2}\left(\Omega_{1}\right)}^{2} \leq \\
\leq & C\left(\left\|v_{0}\right\|_{W_{\varepsilon}^{m, 2 p / 3}\left(\Omega_{2}\right)}^{2 p / 3}+\sum_{i=1}^{3}\left(\left\|v_{i}\right\|_{W_{\varepsilon}^{m, p}\left(\Omega_{2}\right)}^{p}+\left\|v_{i}\right\|_{W^{m-1,2 p}\left(\Omega_{2}\right)}^{2 p}\right)+\right. \\
& \left.+\|w\|_{W_{\varepsilon}^{m, 2 p}\left(\Omega_{2}\right)}^{2 p}+\|v\|_{W_{\varepsilon}^{m, 2 m p}\left(\Omega_{2}\right)}^{2 m p}+\|u\|_{W_{\varepsilon}^{m, 2 m p}\left(\Omega_{2}\right)}^{2 m p}\right)
\end{aligned}
$$

as $\varepsilon \rightarrow 0$.

Proof. This result generalizes Theorem 4.1 in [CLM]. The presence of the term $f$ affects very little the proof of the assertion there.

If $w$ satisfies equation (3.1), by Lemma 2.4 the derivatives of $w$ satisfy an equation of the same type, with different second member, whose coefficients depend on

$$
\frac{\partial_{3} f}{1+\left(\partial_{3} u\right)^{2}} \text { or } \frac{\partial_{3} f \partial_{3} u}{1+\left(\partial_{3} u\right)^{2}} \text {. }
$$

Hence these terms play the same rôle as the coefficients $v_{i}$ of the equation. Thus, as in [CLM] (proof of Th. 4.1 at the end of Sec. 4), we obtain

$$
\begin{aligned}
& \|w\|_{W_{\varepsilon}^{m+1, p}\left(\Omega_{1}\right)}^{p}+\sum_{|I|=m+1}\left\|\left|D_{i} w\right|^{(p-1) / 2}\right\|_{W_{\varepsilon}^{1,2}\left(\Omega_{1}\right)}^{2} \leq \\
\leq & C\left(\left\|v_{0}\right\|_{W_{\varepsilon}^{m, 2 p / 3}\left(\Omega_{2}\right)}^{2 p / 3}+\sum_{i=1}^{3}\left(\left\|v_{i}\right\|_{W_{\varepsilon}^{m, p}\left(\Omega_{2}\right)}^{p}+\left\|v_{i}\right\|_{W^{m-1,2 p}\left(\Omega_{2}\right)}^{2 p}\right)+\|w\|_{W_{\varepsilon}^{m, 2 p}\left(\Omega_{2}\right)}^{2 p}\right. \\
& \left.+\|v\|_{W_{\varepsilon}^{m, 2 p}\left(\Omega_{2}\right)}^{2 p}+\left\|\frac{\partial_{3} f\left(1+\partial_{3} u\right)}{1+\left(\partial_{3} u\right)^{2}}\right\|_{W_{\varepsilon}^{m, p}\left(\Omega_{2}\right)}^{p}+\left\|\frac{\partial_{3} f\left(1+\partial_{3} u\right)}{1+\left(\partial_{3} u\right)^{2}}\right\|_{W^{m-1,2 p}\left(\Omega_{2}\right)}^{2 p}\right) .
\end{aligned}
$$

We obtain the assertion in view of Lemma 3.1. 
In order to apply Theorem 3.2 we have now to study the properties of the function $v=\arctan \left(\partial_{3} u\right)$. A first simple consequence of Lemma 2.4 is the following:

Lemma 3.3 The function $v=\arctan \left(\partial_{3} u\right)$ satisfies the equation

$$
\sum_{j=1}^{3} D_{j}^{2} v=v_{0}+v_{1} D_{1} v+v_{2} D_{2} v
$$

where

$$
\begin{gathered}
v_{0}=\partial_{22} f D_{2} u+\frac{\partial_{3} u \partial_{3} f}{1+\left(\partial_{3} u\right)^{2}}+\frac{\partial_{12} f\left(\partial_{3} u\right)^{2}}{1+\left(\partial_{3} u\right)^{2}}+\frac{\partial_{32} f \partial_{3} u}{1+\left(\partial_{3} u\right)^{2}}-\frac{\partial_{3} u\left(\partial_{3} f\right)^{2}}{1+\left(\partial_{3} u\right)^{2}} \\
v_{1}=-\frac{2 \partial_{3} u \partial_{3} f}{1+\left(\partial_{3} u\right)^{2}}, \quad v_{2}=\frac{\partial_{3} f\left(\left(\partial_{3} u\right)^{2}-1\right)}{1+\left(\partial_{3} u\right)^{2}}
\end{gathered}
$$

Since $u$ is a solution of (3.4), a similar result holds true for $v$ (cf. [CM2], Prop. 3.1). We refer to that paper for the proof.

Lemma 3.4 Let $f \in C^{2}(\Omega)$. For every open set $\Omega_{1} \Subset \Omega$ and $p \geq 1$ there exists a constant $C=C\left(f, p, \Omega_{1}, K\right)$ such that

$$
\left\|\arctan \left(\partial_{3} u\right)\right\|_{W_{\varepsilon}^{1, p}\left(\Omega_{1}\right)} \leq C
$$

as $\varepsilon \rightarrow 0$. The same estimate holds true for $\partial_{3} u$.

By iteration, from Lemma 3.4 we deduce

Proposition 3.5 Let $f \in C^{m+1}(\Omega)$. Let $u=u_{\varepsilon}$ be a solution of (1.9) satisfying (2.38). If $\Omega_{1} \Subset \Omega$ and $p \geq 1$ there exists a constant $C=C\left(f, p, \Omega_{1}, K\right)$ such that

$$
\left\|D_{I} u\right\|_{L^{p}\left(\Omega_{1}\right)}+\left\|\partial_{3}\left(D_{I^{\prime}} u\right)\right\|_{L^{p}\left(\Omega_{1}\right)} \leq C
$$

for every $I$ with $\left|I^{\prime}\right| \leq m,|I| \leq m+1$, and

$$
\left\|D_{I} u\right\|_{L^{2}\left(\Omega_{1}\right)}+\left\|\partial_{3}\left(D_{I^{\prime}} u\right)\right\|_{L^{2}\left(\Omega_{1}\right)} \leq C,
$$

for $\left|I^{\prime}\right|=m+1,|I| \leq m+2$, as $\varepsilon \rightarrow 0$.

Proof. Indeed by (2.38) and Lemma 3.4 there exists a constant $C_{1}$ independent on $\varepsilon \rightarrow 0$ such that

$$
\left\|\left.u\right|_{W_{\varepsilon}^{1, p}\left(\Omega_{1}\right)}+\right\| v \|_{W_{\varepsilon}^{1, p}\left(\Omega_{1}\right)} \leq C_{1} .
$$


Since $u$ is a solution of (1.9) it satisfies (1.10)

$$
\sum D_{j}^{2} u=\partial_{1} f+\partial_{3} f D_{2} u
$$

Then, in view of Theorem 3.2

$$
\|u\|_{W_{\varepsilon}^{2, p}\left(\Omega_{1}\right)} \leq C_{1}
$$

where $C_{1}$ depends only on $f$. Applying the same theorem to $v$ we then obtain

$$
\|u\|_{W_{\varepsilon}^{2, p}\left(\Omega_{1}\right)}+\|v\|_{W_{\varepsilon}^{2, p}\left(\Omega_{1}\right)} \leq C_{1}
$$

and, by iteration,

$$
\|u\|_{W_{\varepsilon}^{m, p}\left(\Omega_{1}\right)}+\|v\|_{W_{\varepsilon}^{m, p}\left(\Omega_{1}\right)} \leq C
$$

for some constant $C$. Moreover, since $u$ belongs to $C^{m}(\Omega)$,

$$
\|u\|_{W_{\varepsilon}^{m+1, p}\left(\Omega_{1}\right)}^{p}+\|u\|_{W_{\varepsilon}^{m+2,2}\left(\Omega_{1}\right)}^{2} \leq C .
$$

The coefficients of the equation satisfied by $v$ are in $C^{m-1}(\Omega)$, which implies

$$
\|v\|_{W_{\varepsilon}^{m, p}\left(\Omega_{1}\right)}^{p}+\|v\|_{W_{\varepsilon}^{m+1,2}\left(\Omega_{1}\right)}^{2} \leq C .
$$

On the other hand, in view of (2.6) and (2.7), we have

$$
\partial_{3} D_{1} u=-\partial_{3} b+\partial_{3} f=\frac{D_{1} \partial_{3} u+\partial_{3} u D_{2} \partial_{3} u}{1+\left(\partial_{3} u\right)^{2}}+\frac{\partial_{3} f}{1+\left(\partial_{3} u\right)^{2}}+\partial_{3} f
$$

Denote $a_{\varepsilon}=a\left(u_{\varepsilon}\right), b_{\varepsilon}=b\left(u_{\varepsilon}\right)$ the coefficients introduced in (1.3) and let $D_{1, \varepsilon}$, $D_{2, \varepsilon}$ be the corresponding vector fields defined in (1.4). Let $X^{j}$ denote any derivative of order $j$ and I a multi-index: $I \in\{1,2,3\}^{m}$. By differentiation and iteration, from the last formula we then have

$$
\partial_{3}\left(D_{I, \varepsilon} u\right)=P\left(\partial_{3} u, X \partial_{3} u, \cdots, X^{m} \partial_{3} u\right)
$$

where $P$ is a rational function whose denominator is a power of $1+\left(\partial_{3} u\right)^{2}$. This concludes the proof.

Corollary 3.6 Let $f \in C^{m+1}(\Omega)$. Let $u$ be a solution of (1.9) satisfying (2.38). For every $\Omega_{1} \Subset \Omega$ and $p \geq 1$ there exists a constant $C=$ $C\left(f, p, \Omega_{1}, \Omega_{2}, K\right)$ such that

$$
\left\|\nabla D_{I^{\prime}} u\right\|_{L^{p}\left(\Omega_{1}\right)}+\left\|\nabla\left(D_{I} u\right)\right\|_{L^{2}\left(\Omega_{1}\right)} \leq C
$$

for every $I$ such that $\left|I^{\prime}\right| \leq m,|I| \leq m+1$, as $\varepsilon \rightarrow 0$. 


\section{Dirichlet problem for the Levi equation}

In this section we prove the existence of a solution of the problem (1.8), letting $\varepsilon$ go to 0 in the approximating problem. In this way we see that the problem has a viscosity solution whose derivatives are in the Sobolev spaces.

\subsection{Existence of a viscosity solution}

Assume that the function $f$ which defines the almost complex structure $J$ is of class $C^{2}$. Given a sequence $\varepsilon_{j} \rightarrow 0$ of positive numbers we denote by $u_{j}$ the solution of the approximated problem: $\mathcal{L}_{\varepsilon_{j}} u_{j}=0$ in $\Omega$ and $u_{j_{\mid b \Omega}}=g$. We also denote by $a_{j}=a\left(u_{j}\right), b_{j}=b\left(u_{j}\right)$, the coefficients introduced in (1.3). Let $D_{\varepsilon_{j}, 1}, D_{\varepsilon_{j}, 2}$ be the corresponding vector fields defined in (1.4), and $D_{\varepsilon_{j}, I}$ the higher order derivatives. By virtue of Theorem 2.7 there exists a constant $C>0$ such that (uniformly with respect to $\varepsilon \rightarrow 0$ )

$$
\left|\nabla_{\varepsilon_{j}} u_{j}\right|^{2} \leq 4\left(\left(D_{\varepsilon_{j}, 1} u_{j}\right)^{2}+\left(D_{\varepsilon_{j}, 2} u_{j}\right)^{2}+\left(\partial_{3} u_{j}\right)^{2}\right) \leq C .
$$

Thus

Theorem 4.1 Let $f$ satisfy the hypothesis of Proposition 2.5. Assume that $b \Omega$ is strictly $J$-pseudoconvex and that the boundary value $g$ is of class $C^{2}$. Then there exists a unique viscosity solution $u \in \operatorname{Lip}(\bar{\Omega})$ of problem (1.8). Moreover

$$
|\nabla u| \leq C
$$

where $C$ is a constant.

Here we adopt the definition of viscosity solution given in [CIL]. The existence immediately follows from the uniform Lipschitz condition (4.1) while the uniqueness can be proved using the technique of [CIL].

\subsection{Regularity properties in the Sobolev spaces}

Let us show that, if $f$ is of class $C^{m+1}$, the solution $u$ belongs to a suitable Sobolev space. The technique of proof here is partially inspired by [CM1] and [CM2].

Following the notations introduced in the previous section let $W_{\varepsilon_{j}}^{k, p}(\Omega)$ be the Sobolev space corresponding to $D_{\varepsilon_{j}, 1}, D_{\varepsilon_{j}, 2}$. By (2.38) and Proposition 3.5 we can assume (changing the sequence $\varepsilon_{j}$ if necessary) that:

$\left\{D_{\varepsilon_{j}, I} u_{j}\right\}$ is strongly convergent in $L_{\text {loc }}^{p}$, weakly in $W_{\text {loc }}^{1, p}(\Omega)$, for every $p>1$ and multi-index $I$ such that $|I| \leq m$;

$\left\{D_{\varepsilon_{j}, I} u_{j}\right\}$ is strongly convergent in $L_{\text {loc }}^{p}$, weakly $W_{\text {loc }}^{1,2}(\Omega)$, for every $p<6$ and multi-index $I$ such that $|I|=m+1$. 
Let

$$
\alpha=\lim _{j \rightarrow \infty} a_{j}, \quad \beta=\lim _{j \rightarrow \infty} b_{j} .
$$

Due to the nonlinearity of $a$ and $b$ as functions of the gradient, it is not obvious that $\alpha=a(u)$, and $\beta=b(u)$ as defined in 1.3 in terms of $u$. This is proved in Lemma 4.2 below. Let us introduce vector fields formally defined as in (1.4), with $\alpha$ and $\beta$ instead of $a$ and $b$ :

$$
\widetilde{D}_{1}=\partial_{1}+\alpha \partial_{3} \quad \widetilde{D}_{2}=\partial_{2}+\beta \partial_{3} .
$$

$\widetilde{D}$ is the gradient $\left(\widetilde{D}_{1}, \widetilde{D}_{2}\right)$ and $W_{0}^{k, p}(\Omega)$ the associated Sobolev space.

Lemma 4.2 . The following holds true:

$\left\{D_{I, \varepsilon_{j}} u_{j}\right\} \rightarrow \widetilde{D}_{I} u$ strongly in $L^{p}$ and weakly in $W_{\text {loc }}^{1, p}(\Omega)$, for every $p>1$ and multi-index $I$ such that $|I| \leq m$;

$\left\{D_{I, \varepsilon_{j}} u_{j}\right\} \rightarrow \widetilde{D}_{I} u$ strongly in $L^{p}$ and weakly in $W_{\mathrm{loc}}^{1,2}(\Omega)$, for every $p<6$ and multi-index $I$ such that $|I|=m+1$.

Proof. Let $\phi$ be a test function. Since $\left\{D_{I, \varepsilon_{j}} u_{j}\right\}$ is convergent in $L_{\text {loc }}^{p}$, we only have to prove that it is weakly convergent to $\widetilde{D}_{I} u$. Integrating by parts

$$
\begin{aligned}
\lim _{j \rightarrow \infty} \int \phi D_{I, \varepsilon_{j}} u_{j} d \lambda= & -\lim _{j \rightarrow \infty} \int u_{j} D_{I, \varepsilon_{j}} \phi d \lambda-\lim _{j \rightarrow \infty} \int u_{j} \phi \partial_{3} a_{j} d \lambda \\
& -\int u D_{I} \phi d \lambda-\int u \phi \partial_{3} a d \lambda
\end{aligned}
$$

and this ensures the weak convergence of $\left\{D_{I, \varepsilon_{j}} u_{j}\right\}$ to $D_{I} u$. The proof for derivatives $D_{I}$ is similar.

As a consequence we derive the expression of $\alpha$ and $\beta$ :

Remark $4.1 \alpha$ and $\beta$ satisfy the following relation:

$$
\alpha=D_{2} u, \quad \beta=-D_{1} u+f,
$$

and

$$
\alpha=a(u)=\frac{\partial_{2} u-\partial_{3} u\left(\partial_{1} u-f\right)}{1+\left(\partial_{3} u\right)^{2}}, \quad \beta=b(u)=-\frac{\partial_{2} u \partial_{3} u+\partial_{1} u-f}{1+\left(\partial_{3} u\right)^{2}}
$$

Proof. Indeed by definition

$$
\alpha=\lim _{j} a_{j}=\lim _{j} D_{\varepsilon_{j}, 2} u_{j}=D_{2} u
$$

while

$$
\beta=\lim _{j} b_{j}=\lim _{j}\left(f-D_{\varepsilon_{j}, 1} u_{j}\right)=f-D_{1} u .
$$


Then

$\alpha=D_{2} u=\partial_{2} u+\beta \partial_{3} u=\partial_{2} u+\left(f-D_{1} u\right) \partial_{3} u=\partial_{2} u+f \partial_{3} u-\left(\partial_{1} u+\alpha \partial_{3} u\right) \partial_{3} u$, $\beta=f-D_{1} u=f-\partial_{1} u-\alpha \partial_{3} u=f-\partial_{1} u-D_{2} u \partial_{3} u=f-\partial_{1} u-\left(\partial_{2} u+\beta \partial_{3} u\right) \partial_{3} u$.

(4.3) immediately follows from these two formulas.

We can now prove the regularity properties of the limit function $u$ in the Sobolev space:

Theorem 4.3 Assume that $f \in C^{m+1}(\Omega)$. Then the function $w=D_{I} u$ belongs to $W_{\text {loc }}^{1, p}(\Omega) \cap W_{0 \text { loc }}^{2,2}(\Omega)$, for every $p>1$ and multi-index $I$ such that $|I| \leq m$. In particular $D_{I} u \in C_{\mathrm{loc}}^{\alpha}$ for every $\alpha<1$ and the equation

$$
D_{1}^{2} u+D_{2}^{2} u=\partial_{1} f+\partial_{3} f D_{2} u
$$

is satisfied everywhere in $\Omega$.

Proof. From Proposition 4.1 we immediately obtain that $D_{0}^{I} u$ belongs to $W_{\text {loc }}^{1, p}(\Omega) \cap W_{0 \text { loc }}^{2,2}(\Omega)$ for every $I,|I| \leq m, p>1$. On the other hand $u_{j}$ is a solution of $\mathcal{L}_{\varepsilon_{j}} u_{j}=0$ for every $j$. Letting $j \rightarrow \infty$, owing to Lemma 4.2, we deduce immediately that $u$ is a solution of (4.4) in the sense of Sobolev spaces. Moreover, by the classical Sobolev embedding theorem, $D_{I} u \in C_{\text {loc }}^{\alpha}(\Omega)$, for all $\alpha<1,|I| \leq m$.

We remark that derivatives $D_{I} u$ here are defined in the sense of distribution. In the next section we prove that in fact they can be computed pointwise.

\section{Existence of Lie derivatives and foliation}

In this section we state regularity properties of the solution $u$ of problem (1.8). As a consequence, through a weak version of Frobenius Theorem, we obtain that the graph of $u$ is foliated by complex curves.

\subsection{A weak version of the Frobenius Theorem}

Let us recall some relations between weak and Lie derivatives, already proved in [CM2]. In that paper a Frobenius theorem for non Lipschitz vector fields was proved, under an analyticity condition in the direction of vector fields. Here we drastically weaken this assumption only requiring that weak derivatives of first order in some directions are bounded, and derivatives in the other directions belong to $L^{p}$.

Let $\Omega \subset \mathbb{R}^{3}, \theta, \zeta \in C_{\text {loc }}^{\alpha}(\Omega), 0 \leq \alpha<1$, and define operators $\widetilde{D}_{1}, \widetilde{D}_{2}$ by

$$
\widetilde{D}_{1}=\partial_{1}+\theta \partial_{3}, \quad \widetilde{D}_{2}=\partial_{2}+\zeta \partial_{3} .
$$


The following remark was proved in [CM2].

Remark 5.1 Assume that $\gamma$ and $\gamma_{1}$ are two solutions of

$$
\left\{\begin{aligned}
\gamma^{\prime} & =\widetilde{D}_{i}(\gamma) \\
\gamma(0) & =\bar{x}
\end{aligned}\right.
$$

with $i$ fixed. There exists a positive constant c such that

$$
d\left(\gamma(s), \gamma_{1}(s)\right)<c s^{1 /(1-\alpha)}
$$

where $d$ is the euclidean distance.

In this situation we say that a function $h$ has the Lie derivative at $\bar{x} \in \Omega$, in the direction of the vector field $\widetilde{D}_{i}$, if, for every solution $\gamma$ of problem (5.1), there exists

$$
\frac{d}{d s}(h \circ \gamma)_{\mid s=0}
$$

and it is independent of $\gamma$.

The weak derivative (in the Sobolev sense) of a sufficiently regular function $h$ coincides with the Lie derivative (cfr. [CM2]):

Proposition 5.1 Let $h \in C^{\beta}(\Omega), \beta>1-\alpha$, with weak derivatives $\widetilde{D}_{i} h, i=$ 1,2 , in $C^{\beta}(\Omega)$. Suppose that $\partial_{3} h \in L_{\mathrm{loc}}^{p}(\Omega), p>1 / \beta$. Then Lie derivatives of $h$ exist for all $x \in \Omega$ and coincide with the weak ones.

We then obtain the following uniqueness theorem

Theorem 5.2 Assume that $\theta, \zeta$ and weak derivatives $\widetilde{D}_{i} \theta, \widetilde{D}_{i} \zeta, i=1,2$ are in $C_{\text {loc }}^{\alpha}(\Omega)$ for $\alpha>1 / 2$, and $\partial_{3} \zeta, \partial_{3} \tau \in L_{\text {loc }}^{p}(\Omega)$ for $p>1 / \alpha$. Then problem (5.1) has a unique solution. In particular, the above notion of Lie derivative coincides with the standard one and the weak derivatives of $\theta$ and $\zeta$ are Lie derivatives.

Proof. Let us consider the problem (5.1), and assume by simplicity that $i=1$. If $\gamma$ is a solution of (5.1), by the definition of the Lie derivative, and Proposition 5.1, the function $\theta \circ \gamma$ is differentiable, with derivative $\widetilde{D}_{1} \theta$. It follows that the coefficient $\theta$ of the equation is of class $C^{1}$ along $\gamma$. If the problem had two solutions $\gamma$ and $\gamma_{1}$, then $\theta$ would be Lipschitz continuous on the union of their graphs, and could be extended to a Lipschitz continuous function on all of $\mathbb{R}^{3}$, by Whitney's theorem. Since $\gamma$ and $\gamma_{1}$ are both solutions of the problem (5.1) with this new second member, they must coincide. The weak derivatives of $\theta$ and $\zeta$ are Lie derivatives by Proposition 5.1. 
Proof of Theorem 1.2 Such a function $t$ exists if and only if $\left(x_{1}, x_{2}\right) \mapsto$ $T\left(x_{1}, x_{2}\right)=\left(x_{1}, x_{2}, t\left(x_{1}, x_{2}\right)\right)$ satisfies

$$
\partial_{1} T\left(x_{1}, x_{2}\right)=(1,0, \theta)\left(t\left(x_{1}, x_{2}\right)\right), \quad \partial_{2} T\left(x_{1}, x_{2}\right)=(0,1, \zeta)\left(t\left(x_{1}, x_{2}\right)\right),
$$

and in that case

$$
T=\exp \left(\left(x_{1}-\bar{x}_{1}\right) \widetilde{D}_{1}+\left(x_{2}-\bar{x}_{2}\right) \widetilde{D}_{2}\right)(\bar{x}) .
$$

Since we have already proved the uniqueness of solutions of problem (5.1), for all $\bar{x}=\left(\bar{x}_{1}, \bar{x}_{2}, \bar{x}_{3}\right)$ in $\Omega$ there exists a neighborhood $B$ of $\left(\bar{x}_{1}, \bar{x}_{2}\right)$ in $\mathbb{R}^{2}$ such that for every $\left(x_{1}, x_{2}\right)$ in $B$ the exponential map is uniquely defined at $\left(x_{1}, x_{2}\right)$. This concludes the proof.

Corollary 5.3 For every function $h=h\left(x_{1}, x_{2}, x_{3}\right)$ of class $C_{\mathrm{loc}}^{\alpha}$ such that $\widetilde{D}_{j} h \in C_{\text {loc }}^{\alpha}, j=1,2$, we have

$$
\begin{aligned}
& \partial_{1} h\left(x_{1}, x_{2}, t\left(x_{1}, x_{2}\right)\right)=\widetilde{D}_{1} h\left(x_{1}, x_{2}, t\left(x_{1}, x_{2}\right)\right), \\
& \partial_{2} f\left(x_{1}, x_{2}, t\left(x_{1}, x_{2}\right)\right)=\widetilde{D}_{2} h\left(x_{1}, x_{2}, t\left(x_{1}, x_{2}\right)\right) .
\end{aligned}
$$

\subsection{Lie derivatives and foliation of the graph}

The proof of Theorem 1.1 is achieved applying Frobenius Theorem to the coefficients $a$ and $b$ of the Levi equation (cfr.1.3).

Theorem 5.4 Assume that $f \in C^{3}(\Omega)$, and let $u$ be a solution of (1.8). Then every $\bar{x} \in \Omega$ lies on a 2-dimensional manifold $S=S_{\bar{x}}$, of class $C^{2}$, such that $u_{\mid S}$ satisfies

$$
\partial_{1}^{2}\left(u_{\mid S}\right)+\partial_{2}^{2}\left(u_{\mid S}\right)=\partial_{1}\left(f_{\mid S}\right)
$$

Proof. We observe that the following conditions are satisfied:

i) $a, b, D_{i} a, D_{i} b \in C^{\alpha}(\Omega)$ for $\alpha>1 / 2$ and $D_{1} b=D_{2} a$,

ii) $\partial_{3} a, \partial_{3} b \in L_{\mathrm{loc}}^{p}(\Omega), p>1 / \alpha$.

Hence we can apply Theorem 1.2: for every fixed $\bar{x} \in \Omega$ there exists a unique solution $t$ of the system

$$
\left\{\begin{array}{l}
\partial_{1} t\left(x_{1}, x_{2}\right)=a\left(x_{1}, x_{2}, t\left(x_{1}, x_{2}\right)\right) \\
\partial_{2} t\left(x_{1}, x_{2}\right)=b\left(x_{1}, x_{2}, t\left(x_{1}, x_{2}\right)\right)
\end{array}\right.
$$

defined in a neighborhood of $\left(\bar{x}_{1}, \bar{x}_{2}\right)$, such that $\bar{x}=\left(\bar{x}_{1}, \bar{x}_{2}, t\left(\bar{x}_{1}, \bar{x}_{2}\right)\right)$. Let us call $T$ be the map $\left(x_{1}, x_{2}\right) \mapsto\left(x_{1}, x_{2}, t\left(x_{1}, x_{2}\right)\right)$, and $S_{\bar{x}}$ its range. Since $D_{I} u \in C^{\alpha}$, for $|I| \leq 2$, then, by Corollary 5.3

$$
\partial_{1}^{2}(u \circ T)+\partial_{2}^{2}(u \circ T)=\partial_{1}(f \circ T) .
$$


Proof of Theorem 1.1 In view of Theorem 4.3 we know that, if $I$ is a multi-index such that $|I| \leq m-1$ and $w=D_{I} u$, then $w$ belongs to $C_{\text {loc }}^{\alpha}$, as well as its derivatives $D_{1} w, D_{2} w$, and $\partial_{3} w \in L_{\text {loc }}^{p}$. By Proposition 5.1 the weak derivatives $D_{i} w$ are Lie derivatives.

In order to prove that the graph of $u$ is foliated by complex curves we need observe that, if $p=(\bar{x}, u(\bar{x})), \bar{x}=\left(\bar{x}_{1}, \bar{x}_{2}, \bar{x}_{3}\right)$, is a point of the graph of $u$ Theorem 5.4 says that $F_{p}=\left\{\left(x,(u \circ T)(x): x \in S_{\bar{x}}\right\}\right.$ is a $C^{2}$ integral variety of the Levi distribution.

\section{References}

[A] Alexander, H.: Polynomial hulls of graphs. Pacif. J. Math 147 (1991), 201-212.

[BG] Bedford, E. ANd Gaveau, B.: Envelopes of holomorphy of certain 2-spheres in $\mathbb{C}^{2}$. Am. J. Math. 105 (1983), 975-1009.

[BK] Bedford, E. And Klingenberg, W.: On the envelope of holomorphy of a 2-sphere in $\mathbb{C}^{2}$. J. Amer. Math. Soc. 4 (1991), 623-646.

[CS] Chirka, E. M. and Shcherbina, N.: Pseudoconvexity of rigid domains and foliations of hulls of graphs. Ann. Scuola Norm. Sup. Pisa Cl. Sci. (4) 21 (1995), 707-735.

[CM1] Citti, G. And Montanari, A.: Strong solutions for the Levi curvature equation. Adv. Differential Equations 5 (2000), no. 1-3, 323-342.

[CM2] Citti, G. and Montanari, A.: Analytic estimates for solutions of the Levi equation. J. Differential Equations 173 (2001), 356-389.

[CLM] Citti, G., Lanconelli, E. and Montanari, A.: Smoothness of Lipschitz continuous graphs with nonvanishing Levi curvature. Acta Math. 188 (2002), 87-128.

[CIL] Crandall, M. G., Ishit, H. and Lions, P. L.: User's guide to viscosity solutions of second order partial differential equations. Bull. Amer. Math Soc. (N.S.) 27 (1992), 1-67.

[F] Folland, G. B.: Subelliptic estimates and function on nilpotent Lie groups. Ark. Mat. 13 (1975), 161-207.

[FS] Folland, G. B. And Stein, E.: Estimates for the $\bar{\partial}_{b}$ complex and analysis on the Heisenberg Group. Comm. Pure Appl. Math. 27 (1974), 429-522.

[FL] Franchi, B. AND LANCONELli, E.: Hölder regularity theorem for a class of linear nonuniformly elliptic operators with measurable coefficients. Ann. Scuola Norm. Sup. Pisa Cl. Sci. (4) 10 (1983), 523-541.

[G] Gromov, M.: Pseudo-holomorphic curves in symplectic manifolds. Invent. Math. 82 (1985), 307-347.

[K] KruZ̆ILIN, N. G.: Two-dimensional spheres on the boundaries of pseudoconvex domains in $\mathbb{C}^{2}$. Izv. Akad. Nauk SSSR Ser. Mat. 55 (1991), 11941237. 
[RS] Rothschild, L. And Stein, E. M.: Hypoelliptic differential operators and nilpotent groups. Acta Math. 137 (1977), 247-320.

[S] Shcherbina, N.: On the polynomial hull of a graph. Indiana Univ. Math. J. 42 (1993), 477-503.

[ST] Shcherbina, N. And Tomassini, G.: The Dirichlet problem for Levi flat graphs over unbounded domains. Internat. Math. Res. Notices no. 3 (1999), $111-151$.

[SIT] Slodkowski, Z. And Tomassini, G.: Weak solutions for the Levi equation and envelope of holomorphy. J. Funct. Anal. 101 (1991), no. 2, 392-407.

Recibido: 17 de abril de 2002

Revisado: 19 de julio de 2002

Giovanna Citti

Dipartimento di Matematica

Università di Bologna

Piazza di Porta S. Donato 5

40127 Bologna, Italy

citti@dm.unibo.it

Giuseppe Tomassini

Scuola Normale Superiore di Pisa

Piazza dei Cavalieri 7

56126 Pisa, Italy

tomassini@sns.it

Supported by University of Bologna "Founds for selected research topics". Supported by the project MURST "Geometric Properties of Real and Complex Manifolds". 